\title{
Tuning of subwavelength topological interface states in locally resonant metastructures with shunted piezoelectric patches ${ }^{\text {a) }}$
}

\author{
Yijie Liu, ${ }^{1, b)}$ Wenbo Fang, ${ }^{1}$ Yingjing Liang, ${ }^{1}$ Dianzi Liu, ${ }^{2}$ and Qiang $\mathrm{Han}^{3}$ \\ 1) School of Civil Engineering, Guangzhou University, Guangzhou, 510006, P.R. China \\ 2) University of East Anglia, Norwich, NR47TJ, UK \\ ${ }^{3)}$ School of Civil Engineering and Transportation, South China University of Technology, Guangzhou, 510600, \\ P.R.China
}

(Dated: 9 June 2021)

We investigate the propagation behavior of the low-frequency topological interface state of the flexural wave in the locally resonant metastructure and analyze the tunability of the sub-wavelength interface states by the piezoelectric shunting circuit. One homogeneous thin beam is periodically attached with local resonant beams, which connect shunted piezoelectric actuators. The folding band obtained by merging two primitive unit cells into one new element can generate a Dirac point below the low-frequency locally resonant bandgap. This folding point is opened to develop one new bandgap originated from the Bragg scattering effect by breaking the mirror symmetry. Then, topological transitions are demonstrated during the distance variation between two adjacent resonances. The interface state's existence is further confirmed by using steady and transient analysis of the heterostructure, composed of two media with different topological properties. Finally, we show the relationship between the interface frequency and the capacitance ratio and research the influence of the distance parameter on the topological interface state. Because of the tunability of elastic waves by the piezoelectric shunting circuit, our design has potential for applications such as energy harvesters, filters, and physical switches.

Keywords: Tunable topological interface state, Sub-wavelength flexural wave, Local resonant metastructures, Shunted piezoelectric patches

\section{INTRODUCTION}

Topological insulators (TIs), originally associated with quantum state theory and condense matter, have been adopted in acoustics and optics in recent years. Many mimic quantum topological characteristic, such as the quantum Hall effect ${ }^{1,2}$, quantum spin Hall effect ${ }^{3-5}$, and quantum valley Hall effect ${ }^{6-8}$ have also been realized in mechanical elastic systems. Among these, topologically protected interface (edge) states (TPEs) ${ }^{9,10}$ in elastic insulator materials have gained particular interest. Elastic TIs allow wave propagation only at the interface/edge ${ }^{11}$. Hence, TPEs are immune to back scattering from defects or disorders, allowing to precisely guide and control the wave propagation path ${ }^{12,13}$. Elastic TIs could be employed to design a variety of high performance acoustic devices, with a wide range of applications communication, sensing and nondestructive testing ${ }^{14,15}$.

In electron and photon systems, the time-reversal symmetry is easily broken by using the magnetic field, resulting in a quantum Hall topological insulator. However, the most difficult challenge in phonon systems is in terms of specimen preparation and experimental operation of the applied external field as it breaks the time-reversal symmetry $^{16}$. Huber et al. ${ }^{17}$ generated quantum Hall effect-like features to obtain spiral topological edge states

\footnotetext{
a) Journal of Applied Physics

b) Electronic mail: celiuyijie@gzhu.edu.cn; School of Civil Engineering, Guangzhou University, Guangzhou, 510006, P.R.China
}

by using mechanical oscillators. Wang et al. obtained phonon edge states with defect immunity by breaking the spatial mirror symmetry to generate the quantum spin Hall effect ${ }^{18}$. Analogously, the quantum Hall valley effect can be realized by applying passive factors to break the mirror symmetry of the $C_{3 v}$ system $^{19-21}$, as they keep time-reversal symmetry intact. These elastic metastructures are constructed to generate a Dirac cone at the high symmetry point in the reduced Brillouin zone. Breaking the mirror symmetry results in opening the Dirac points, which creates two kinds of band-gaps with different topological properties. The double Dirac cone emerges in the $C_{6 v}$ symmetry system ${ }^{22,23}$ due to the zone-folding of the frequency band structure, which is opened to obtain the Hall pseudo-spin TI. In phononic crystals, one-dimensional topological interface states exhibit a fixed frequency value in a certain range.

In previous studies they usually display high frequency and narrow bandwidths. Therefore, it is desirable to have a tunable phononic crystal to operate at various frequency domains and adapt to changes in the environment. The bandgap and topological properties can be tuned by applying an external load ${ }^{24-26}$, a thermal field $^{27}$, an electric field ${ }^{22,28}$ or a magnetic field ${ }^{29}$. The piezoelectric shunt circuit has the advantage of being light weight, small, easy to install and fabricate, and precise controllability $^{30}$. Airoldi and Ruzzene proposed one periodic array of piezoelectric patches with electrodes attached to a resonant electric circuit to obtain the tuning frequency of the shunting circuits ${ }^{31}$, and also considered elastic wave propagation in fluid-loaded shells with periodic shunted piezoelectric rings ${ }^{32}$. Then, Spadoni et al. 
constructed the two-dimensional periodic piezoelectrical meta-plate to analyze wave propagation control of the shunted circuit ${ }^{33}$, which was confirmed experimentally ${ }^{34}$. Zhou et al. proposed a periodic piezoelectric $\operatorname{rod}^{35}$ and beam metastructure ${ }^{36}$ to obtain tunable topological interface states for longitudinal and flexural elastic waves via employing electrical shunting circuits, respectively. The locally resonant sub-structure can be utilized to realize the lower frequency bandgap in the sub-wavelength region. One novel locally resonant meta-beam was proposed by Xiao et al. ${ }^{37}$ employing periodic arrays of beamlike resonators attached to a base beam, obtaining local resonant and Bragg band gaps. Inspired by the former model ${ }^{37,38}$, Zhou et al. ${ }^{39}$ utilized the piezoelectrical shunt circuit to control the stiffness of the beam-like resonator to tune the local resonant bandgap at a very low frequency region, which can reduce the attached mass, whilst keeping the integrity of the original structure.

In this work, the this beam-like locally resonant metastructure is employed to realize the sub-wavelength topological interface state. We also utilized the shunted piezoelectric patch to tune the interface frequency of flexural waves to adapt to the changing external environment. This paper is organized as follows. The beam-like local resonant metastructure model with shunted piezoelectric patches is introduced in Section 2. Section 3 presents the spectral element formulation of this metastructure, which can be employed to calculate band structures. We analyze the band evolution by using the spectral element method in Section 4 to consider the existence of the topological transition during the distance variation between the axes of the two beam-like resonators. Finally, Section 5 numerically investigates the behavior of the topological interface state in the heterostructure and discusses the piezoelectric shunting circuit's tunable ability on the sub-wavelength interface states.

\section{LOCALLY RESONANT METASTRUCTURE MODEL WITH SHUNTED PIEZOELECTRIC PATCHES}

The locally resonant metastructure presented in this paper has consisted of four parts: base beam, connector, mass block, and piezoelectric bimorph beam, as shown in Fig. 1, whose tunable dynamical behaviors in terms of wave propagation are considered through theoretical analysis and numerical simulations. This bimorph structure with a rectangular cross-section is made from two continuous piezoelectric layers sandwiching a central epoxy shim. The negative capacitance circuits connected at the bimorph beam are employed to control the equivalent bending stiffness to change the propagation behavior of elastic waves. Besides, its geometrical properties are given in Table. I. Assuming that the piezo sheet is only subjected to the electric field along the $z$-direction. All surfaces on the piezo sheet are in the stress-free state except for the cross-sections perpendicular to the $x$-axis. Then, the stress components $\sigma_{j}(j=2,3,4,5,6)$ equal approximately zero. Hence, the constitutive equation of the piezoelectric layer can be simplified as ${ }^{40,41}$

$$
\left[\begin{array}{l}
\mathcal{S}_{1} \\
\mathcal{D}_{3}
\end{array}\right]=\left[\begin{array}{ll}
s_{11}^{E} & d_{31} \\
d_{31} & \varepsilon_{33}^{T}
\end{array}\right]\left[\begin{array}{c}
\sigma_{1} \\
E_{3}
\end{array}\right]
$$

where $\mathcal{S}_{1}$ and $\sigma_{1}$ denote the strain and stress components along the $x$-axis, respectively. $\mathcal{D}_{3}$ and $E_{3}$ describe the electrical displacement and electrical field along the $z$-direction, respectively. $s_{11}^{E}$ is the elastic compliance at constant electric field, $d_{31}$ is the piezoelectric strain constant, $\varepsilon_{33}^{T}$ is the permittivity component at constant stress.

In this paper, the primary negative capacitance shunt circuit is used to tune the equivalent bending stiffness of the bimorph beam, and its complex impedance is expressed as

$$
Z=\frac{1}{s C_{N}}
$$

where $C_{N}$ denotes the negative capacitance. $s$ is the Laplace domain complex variable, expressed by $i \omega$, where $i$ and $\omega$ denote the imaginary unit and the angular frequency, respectively. According to the long wave approximation method, the electric current in the shunt circuit can be expressed as

$$
I=\frac{U}{Z}=-s \mathcal{D}_{3} A_{s}
$$

where $U=E_{3} h_{p}$ is the voltage between the upper and lower electrodes of the piezo sheet and $A_{s}$ is the area of the piezoelectric patch electrodes. $h_{p}$ denotes the thickness of the piezo sheet. The substitution of Eq. (3) into Eq. (1) yields

$$
\mathcal{S}_{1}=\left(s_{11}^{E}-\frac{s Z d_{31}^{2} A_{s} h_{p}}{1+s Z C_{P}^{S}}\right) \sigma_{1}
$$

The effect of electric shunting on the piezo sheet enhances the piezo sheet's equivalent bending stiffness. From Eq. (4), the equivalent elastic modulus of this sheet can be given as

$$
E_{p}=\frac{1}{s_{11}^{E}}\left[1+\frac{s Z d_{31}^{2} A_{s}}{s_{11}^{E} h_{p}\left(1+s Z C_{P}^{S}\right)}\right]
$$

Before analyzing the overall metastructure, the local resonance is studied, which combines the end mass block, the bimorph beam, and the connector, shown in Fig. 1(b). The connector is fixed at the base beam, regarded as a rigid mass block. The composite structure made from the end mass block and bimorph beam can be simplified as a cantilever beam structure, which can be investigated using the Euler-Bernoulli beam model. The governing equation for this composite cantilever beam can be defined as

$$
D(y) \frac{\partial^{4} w(y, t)}{\partial y^{4}}+G(y) \frac{\partial^{2} w(y, t)}{\partial t^{2}}=0
$$




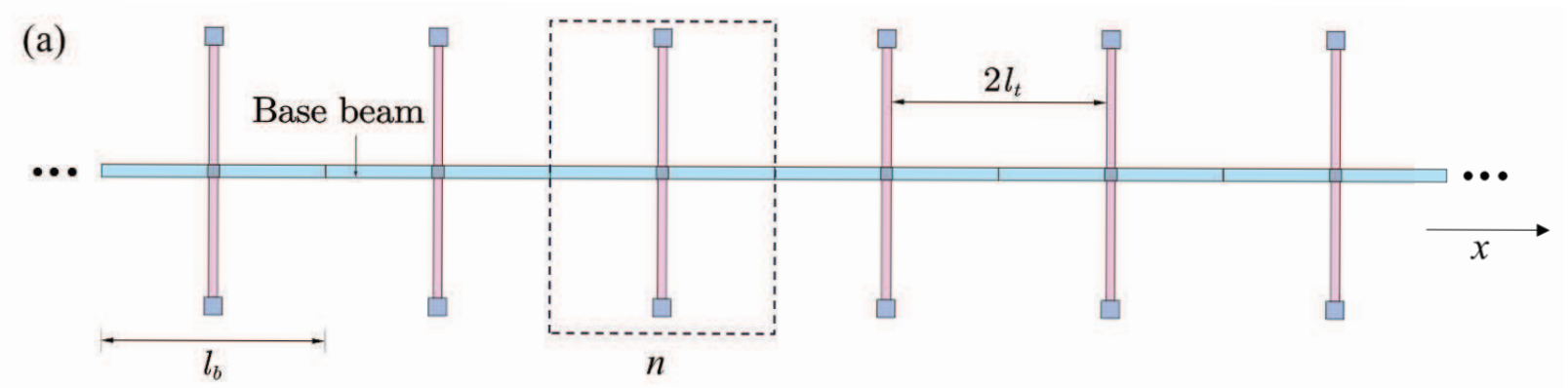

(b)

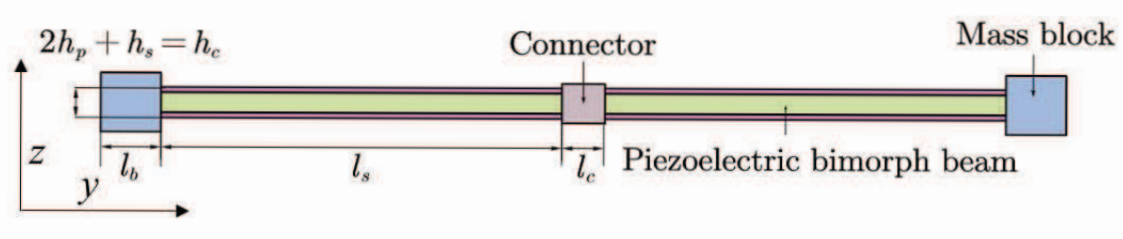

(c)

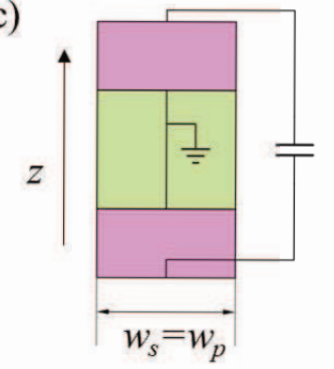

FIG. 1: The schematic map of the locally resonant metastructure model with shunted piezoelectric patches: (a) The metastructure model; (b) The piezoelectric bimorph beam; (c) The piezoelectric shunting circuits.

TABLE I: The geometrical parameters of the locally resonant metastructure.

\begin{tabular}{lccc}
\hline Component & Length $(\mathrm{mm})$ & Width $(\mathrm{mm})$ & Height $(\mathrm{mm})$ \\
\hline Sandwich beam & 75 & 10 & 2.5 \\
Piezoelectric layer & 75 & 10 & 0.25 \\
Connector & 6 & 10 & 3 \\
Base beam & 100 & 10 & 2 \\
Mass & 6 & 10 & 6 \\
\hline
\end{tabular}

with

$$
\begin{gathered}
D(y)=2 E_{p} I_{p}+E_{s} I_{s} \\
G(y)=2 \rho_{p} A_{p}+2 \rho_{s} A_{s}
\end{gathered}
$$

where $D(y)$ represents the bending stiffness of the bimorph beam, $G(y)$ denotes the equivalent density per unit length. $E_{s}$ and $E_{p}$ are the elastic modulus of the epoxy shim and piezo sheet, respectively. Piezo sheets exhibit different levels of elasticity stiffness in different circuit environments. $I_{s}$ and $I_{p}$ are the inertia moments of the epoxy shim and piezo sheet, respectively. $A_{s}$ and $A_{p}$ are the cross-sectional areas of the epoxy shim and piezo sheet, respectively. $\rho_{s}$ and $\rho_{p}$ are the epoxy shim and piezo sheet density, respectively.

Generally, the steady-state solution of the above bend- ing equation can be expressed as

$$
\begin{aligned}
w(y, t) & =W(y) e^{j \omega t}=\left[C_{1} \cos \left(\kappa_{1} y\right)+C_{2} \sin \left(\kappa_{1} y\right)\right. \\
& \left.+C_{3} \cosh \left(\kappa_{1} y\right)+C_{4} \sinh \left(\kappa_{1} y\right)\right] e^{j \omega t}
\end{aligned}
$$

with

$$
\kappa_{1}=\sqrt[4]{\frac{G \omega^{2}}{D}}
$$

where $C_{1}, C_{2}, C_{3}$ and $C_{4}$ are undetermined constants. By utilizing the boundary continuity of the vibration and rotation of the mass block, the initial conditions of the 
differential equation Eq. (8) are described as

$$
\begin{gathered}
\left.D \frac{\partial^{3} W(y)}{\partial y^{3}}\right|_{y=0}=F_{0} \\
\left.\frac{\partial W(y)}{\partial y}\right|_{y=0}=0 \\
\left.D \frac{\partial^{3} W(y)}{\partial y^{3}}\right|_{y=l_{f}}=-\omega^{2} m_{M} W\left(l_{f}\right) \\
\left.D \frac{\partial^{2} W}{\partial y^{2}}\right|_{l_{f}}+\left.\frac{D l_{M}}{2} \frac{\partial^{3} W}{\partial y^{3}}\right|_{l_{f}}=\left.\omega^{2} J_{M} \frac{\partial W}{\partial y}\right|_{l_{f}}
\end{gathered}
$$

where $F_{0}$ is the interaction between the base beam and the connector. $J_{M}$ and $m_{M}$ denote the rotational inertia and mass of the end block, respectively, which can be given as

$$
m_{M}=\rho_{M} h_{M} b_{M} l_{M}
$$

and

$$
J_{M}=\frac{1}{12} \rho_{M} h_{M} b_{M} l_{M}\left(h_{M}^{2}+l_{M}^{2}\right)
$$

After substituting Eq. (9) into Eq. (11), the coefficients can be given as

$$
\begin{aligned}
C_{1} & =\frac{\xi_{1} A_{1}+\xi_{2} A_{2}+\xi_{3} A_{3}+\xi_{4} A_{4}+\xi_{5} A_{5}+\xi_{6} A_{6}}{2 D \kappa_{1}^{2}\left[\left(\xi_{7} A_{2}-\xi_{2} A_{5}\right)+\left(\xi_{2} A_{3}+\xi_{5} A_{4}\right)\right]} \\
C_{2} & =-\frac{F_{0}}{2 D \kappa_{1}^{3}} \\
C_{3} & =\frac{\xi_{8}\left(A_{1}+A_{7}\right)+\xi_{5} A_{3}+\xi_{2} A_{4}-\xi_{4} A_{2}+\xi_{7} A_{5}}{2 D \kappa_{1}^{2}\left[\left(\xi_{7} A_{2}-\xi_{2} A_{5}\right)+\left(\xi_{2} A_{3}+\xi_{5} A_{4}\right)\right]} \\
C_{4} & =\frac{F_{0}}{2 D \kappa_{1}^{3}}
\end{aligned}
$$

with

$$
\begin{aligned}
& \xi_{1}=-2 D^{2} \kappa_{1}^{4}+D \kappa_{1}^{2} l_{M} m_{M} \omega^{2}-2 J_{M} m_{M} \omega^{4} \\
& \xi_{2}=4 D \kappa_{1} m_{M} \omega^{2} \\
& \xi_{3}=-2 D^{2} \kappa_{1}^{4}+D \kappa_{1}^{2} l_{M} m_{M} \omega^{2}+2 J_{M} m_{M} \omega^{4} \\
& \xi_{4}=4 D J_{M} \kappa_{1}^{3} \omega^{2} \\
& \xi_{5}=2 D^{2} \kappa_{1}^{4}+D \kappa_{1}^{2} l_{M} m_{M} \omega^{2}-2 J_{M} m_{M} \omega^{4} \\
& \xi_{6}=2 D^{2} \kappa_{1}^{4}-D \kappa_{1}^{2} l_{M} m_{M} \omega^{2}+2 J_{M} m_{M} \omega^{4} \\
& \xi_{7}=2 D^{2} \kappa_{1}^{4}-D \kappa_{1}^{2} l_{M} m_{M} \omega^{2}-2 J_{M} m_{M} \omega^{4} \\
& \xi_{8}=2 D^{2} \kappa_{1}^{4}+D \kappa_{1}^{2} l_{M} m_{M} \omega^{2}+2 J_{M} m_{M} \omega^{4} \\
& A_{1}=\cosh \left(\kappa_{1} l_{s}\right), A_{2}=\sin \left(\kappa_{1} l_{s}\right) \cosh ^{2}\left(\kappa_{1} l_{s}\right) \\
& A_{3}=\cos \left(\kappa_{1} l_{s}\right) \cosh \left(\kappa_{1} l_{s}\right), A_{7}=\sin ^{2}\left(\kappa_{1} l_{s}\right) \\
& A_{5}=\sin \left(\kappa_{1} l_{s}\right) \sinh \left(\kappa_{1} l_{s}\right), A_{6}=\sinh ^{2}\left(\kappa_{1} l_{s}\right) \\
& A_{4}=\cos \left(\kappa_{1} l_{s}\right) \sinh \left(\kappa_{1} l_{s}\right)
\end{aligned}
$$

Obviously, the dynamic stiffness of the cantilever beam is given as

$$
S_{f}=\frac{F_{0}}{W(0)}=\frac{F_{0}}{C_{1}+C_{3}}
$$

Besides, the dynamic stiffness of the connector can also be simplified as

$$
S_{c}=-m_{c} \omega^{2}
$$

where $m_{c}$ denotes the mass of the connector. Hence, the total dynamic stiffness of the combination of mass block, bimorph beam, and connector can be expressed as

$$
S_{t}=2 S_{f}+S_{c}
$$

And, the relationship between the force $F$ and deflection $\bar{w}_{j}$ at the junction of this metastructure is described as

$$
F=S_{t} \bar{w}_{j}
$$

The deflection can be regarded as a disturbance in the connection between the base beam and the additional structure.

Similarly, the vibration of the base beam can be described by the Euler-Bernoulli beam theory. The governing equation of the base beam can be given as

$$
E_{b} I_{b} \frac{\partial^{4} \bar{w}(x, t)}{\partial x^{4}}+\rho_{b} A_{b} \frac{\partial^{2} \bar{w}(x, t)}{\partial t^{2}}=0
$$

where $\rho_{b}, E_{b} I_{b}$ and $A_{b}$ are the density. the bending stiffness, and the cross-sectional area of the base beam, respectively. $\quad E_{b}$ and $I_{b}$ denote the elastic modulus and inertia moment of the base beam, respectively. Assuming $\bar{w}(x, t)=\bar{W}(x) e^{i \omega t}$, the solution of the flexural wave for the base beam can be described as

$$
\begin{aligned}
\bar{W}(x)= & C_{5} \cos \left(\kappa_{b} x\right)+C_{6} \sin \left(\kappa_{b} x\right) \\
& +C_{7} \cosh \left(\kappa_{b} x\right)+C_{8} \sinh \left(\kappa_{b} x\right)
\end{aligned}
$$

with

$$
\kappa_{b}=\sqrt[4]{\frac{\rho_{b} A_{b} \omega^{2}}{E_{b} I_{b}}}
$$

where $C_{5}, C_{6}, C_{7}$ and $C_{8}$ are undetermined constants.

\section{THE SPECTRAL ELEMENT FORMULATION WITH THE DISPERSIVE RELATIONSHIP}

One primitive cell is extracted from the periodically aligned structure, whose schematic representation is given in the dashed box of Fig. 1(a). $\mathbf{q}_{L}$ and $\mathbf{q}_{R}$ denote the displacement vectors at the left and right boundary, respectively. $\mathbf{f}_{L}$ and $\mathbf{f}_{R}$ are the load vectors at the left and right boundary, respectively. The subscripts $L$ and $R$ represent the left and right ends of the cell model, respectively. In the Euler-Bernoulli model, the above displacement and load vectors also are given as

$$
\mathbf{q}_{L}=\left[\begin{array}{c}
u_{L} \\
\theta_{L}
\end{array}\right], \mathbf{f}_{L}=\left[\begin{array}{c}
F_{L} \\
M_{L}
\end{array}\right]
$$


and

$$
\mathbf{q}_{R}=\left[\begin{array}{l}
u_{R} \\
\theta_{R}
\end{array}\right], \mathbf{f}_{R}=\left[\begin{array}{l}
F_{R} \\
M_{R}
\end{array}\right]
$$

where $\theta$ is the slope, and $u$ is the transverse displacement. $Q$ and $M$ denote the shear force and bending moment, respectively. The relationship between the load and displacement vectors can be described as

$$
\begin{aligned}
\theta(x) & =\bar{W}^{\prime}(x), M(x)=E_{b} I_{b} \bar{W}^{\prime \prime}(x) \\
F(x) & =-E_{b} I_{b} \bar{W}^{\prime \prime \prime}(x)
\end{aligned}
$$

In the framework of the spectral element method, the dynamic stiffness of the metastructure beam can be rewritten as

$$
\mathbf{D}_{\text {beam }}=\left[\begin{array}{ll}
\mathbf{D}_{11} & \mathbf{D}_{12} \\
\mathbf{D}_{21} & \mathbf{D}_{22}
\end{array}\right]
$$

The submatrices within the dynamic stiffness matrix of Eq. (23) can be expressed as

$$
\begin{aligned}
& \mathbf{D}_{11}\left(x=l_{b}\right)=\frac{E_{b} I_{b}}{x^{3}}\left[\begin{array}{cc}
\alpha & \bar{\gamma} x \\
\bar{\gamma} x & \beta x^{2}
\end{array}\right] \\
& \mathbf{D}_{12}\left(x=l_{b}\right)=\frac{E_{b} I_{b}}{x^{3}}\left[\begin{array}{cc}
-\bar{\alpha} & \gamma x \\
-\gamma x & \bar{\beta} x^{2}
\end{array}\right] \\
& \mathbf{D}_{21}\left(x=l_{b}\right)=\frac{E_{b} I_{b}}{x^{3}}\left[\begin{array}{cc}
-\bar{\alpha} & -\gamma x \\
\gamma x & \bar{\beta} x^{2}
\end{array}\right] \\
& \mathbf{D}_{22}\left(x=l_{b}\right)=\frac{E_{b} I_{b}}{x^{3}}\left[\begin{array}{cc}
\alpha & -\bar{\gamma} x \\
-\bar{\gamma} x & \beta x^{2}
\end{array}\right]
\end{aligned}
$$

where the components of these submatrixs are given as

$$
\begin{aligned}
\alpha & =\left[\cos \left(\kappa l_{b}\right) \sinh \left(\kappa l_{b}\right)+\sin \left(\kappa l_{b}\right) \cosh \left(\kappa l_{b}\right)\right]\left(\kappa l_{b}\right)^{3} / \Delta \\
\bar{\alpha} & =\left[\sin \left(\kappa l_{b}\right)+\sinh \left(\kappa l_{b}\right)\right]\left(\kappa l_{b}\right)^{3} / \Delta \\
\beta & =\left[-\cos \left(\kappa l_{b}\right) \sinh \left(\kappa l_{b}\right)+\sin \left(\kappa l_{b}\right) \cosh \left(\kappa l_{b}\right)\right] \kappa l_{b} / \Delta \\
\bar{\beta} & =\left[-\sin \left(\kappa l_{b}\right)+\sinh \left(\kappa l_{b}\right)\right] \kappa l_{b} / \Delta \\
\gamma & =\left[-\cos \left(\kappa l_{b}\right)+\cosh \left(\kappa l_{b}\right)\right]\left(\kappa l_{b}\right)^{2} / \Delta \\
\bar{\gamma} & =\sin \left(\kappa l_{b}\right) \sinh \left(\kappa l_{b}\right)\left(\kappa l_{b}\right)^{2} / \Delta \\
\Delta & =1-\cos \left(\kappa l_{b}\right) \cosh \left(\kappa l_{b}\right)
\end{aligned}
$$

The dynamic stiffness of the local resonance can be regarded as the application at the left end of the base beam. Consequently, the motion equation in the spectral approach yields ${ }^{42,43}$

$$
\left[\begin{array}{ll}
\mathbf{D}_{L L} & \mathbf{D}_{L R} \\
\mathbf{D}_{R L} & \mathbf{D}_{R R}
\end{array}\right]\left[\begin{array}{l}
\mathbf{q}_{L} \\
\mathbf{q}_{R}
\end{array}\right]=\left[\begin{array}{l}
\mathbf{f}_{L} \\
\mathbf{f}_{R}
\end{array}\right]
$$

with

$$
\begin{aligned}
& \mathbf{D}_{L L}=\mathbf{D}_{11}+\mathbf{D}_{f}, \quad \mathbf{D}_{L R}=\mathbf{D}_{R L}^{\mathrm{T}}=\mathbf{D}_{12} \\
& \mathbf{D}_{R R}=\mathbf{D}_{22}, \quad \mathbf{D}_{t}=\left[\begin{array}{cc}
S_{t} & 0 \\
0 & 0
\end{array}\right]
\end{aligned}
$$

Based on the Bloch theory, the relationships associated with the field variables on the two ends of the cell are expressed as

$$
\mathbf{q}_{R}=e^{i k l_{b}} \mathbf{q}_{L}, \mathbf{f}_{R}=-\mathbf{f}_{L} e^{i k l_{b}}
$$

where $l_{b}$ denotes the length of the primitive cell. $k$ is the Bloch parameter associated with the wavenumber. $i k l_{b}$ can be collectively referred to as a periodic propagation constant later in the paper. The real and imaginary parts of $i k l_{b}$ denote the attenuation and phase constants, respectively.

Combining Eq. (30) with Eq. (28), the dispersion relationship of this metastructure can be expressed as

$$
\cos ^{2}\left(k l_{b}\right)+\alpha_{1} \cos \left(k l_{b}\right)+\alpha_{2}=0
$$

with

$$
\begin{aligned}
\alpha_{1}= & -\left[\cos \left(\kappa l_{b}\right)+\cosh \left(\kappa l_{b}\right)\right] \\
& -\frac{S_{f}}{4 E_{b} I_{b} \kappa}\left[\sin \left(\kappa l_{b}\right)-\sinh \left(\kappa l_{b}\right)\right]
\end{aligned}
$$

$$
\begin{aligned}
& \alpha_{2}=\cos \left(\kappa l_{b}\right) \cosh \left(\kappa l_{b}\right)+ \\
& \frac{S_{f}}{4 E_{b} I_{b} \kappa}\left[\sinh \left(\kappa l_{b}\right) \cosh \left(\kappa l_{b}\right)-\cos \left(\kappa l_{b}\right) \sinh \left(\kappa l_{b}\right)\right]
\end{aligned}
$$

We can easily check that Eq. (32) is a quadratic equation for $\cos \left(k l_{b}\right)$, which has two different propagation constants at each given frequency in $0 \leqslant k l_{b} \leqslant \pi$. Due to the $k$ axis symmetry of $\cos \left(k l_{b}\right)$, both $k l_{b}$ and $-k l_{b}$ are solutions of Eq. (32), which perform the same wave propagation property but just only travel in opposite directions. Generally, the propagation constants may be purely real, purely imaginary, or complex. Purely real wavenumbers correspond to propagation modes without attenuation under no damp, while purely imaginary wavenumbers called evanescent modes are regarded as the perturbance. All others with full complex eigenvalue represent inhomogenous modes, decaying exponentially in the propagation direction. Here, the propagation modes are only considered to study the dynamic behavior of the metastructure.

In this paper, the band-folding mechanism is employed to realize the subwavelength topological state with a very low frequency. We will merge two primitive cells into a new element and essentially give the dynamic spectral element formulation of this new metastructure. The dynamic equation of the new cell can be written as

$$
\left[\begin{array}{ccc}
\overline{\mathbf{D}}_{L L} & \overline{\mathbf{D}}_{L I} & 0 \\
\overline{\mathbf{D}}_{I L} & \overline{\mathbf{D}}_{I I} & \overline{\mathbf{D}}_{I R} \\
0 & \overline{\mathbf{D}}_{R I} & \overline{\mathbf{D}}_{R R}
\end{array}\right]\left[\begin{array}{c}
\mathbf{q}_{L} \\
\mathbf{q}_{I} \\
\mathbf{q}_{R}
\end{array}\right]=\left[\begin{array}{c}
\mathbf{f}_{L} \\
\mathbf{0} \\
\mathbf{f}_{R}
\end{array}\right]
$$

with

$$
\begin{aligned}
& \overline{\mathbf{D}}_{L L}=\mathbf{D}_{11}\left(x=l_{1}\right)+\mathbf{D}_{f} \\
& \overline{\mathbf{D}}_{I R}=\overline{\mathbf{D}}_{R I}^{T}=\mathbf{D}_{12}\left(x=l_{2}\right) \\
& \overline{\mathbf{D}}_{I I}=\mathbf{D}_{22}\left(x=l_{1}\right)+\mathbf{D}_{11}\left(x=l_{2}\right)+\mathbf{D}_{f} \\
& \overline{\mathbf{D}}_{L I}=\overline{\mathbf{D}}_{I L}^{T}=\mathbf{D}_{12}\left(x=l_{1}\right), \overline{\mathbf{D}}_{R R}=\mathbf{D}_{22}\left(x=l_{2}\right)
\end{aligned}
$$


where $l_{1}$ and $l_{2}$ described the length of the first and second parts, respectively. $\mathbf{q}_{I}$ is the displacement of the connection between two primitive parts. Note that $\mathbf{q}_{I}$ can be rewritten from Eq. (34) as

$$
\mathbf{q}_{m}=-\overline{\mathbf{D}}_{I I}^{-1}\left(\overline{\mathbf{D}}_{I L} \mathbf{q}_{L}+\overline{\mathbf{D}}_{I R} \mathbf{q}_{R}\right)
$$

After substituting Eq. (36) into Eq. (34), the dynamic spectral element matrix can be reconstructed as

$$
\left[\begin{array}{ll}
\mathbf{S}_{L L} & \mathbf{S}_{L R} \\
\mathbf{S}_{R L} & \mathbf{S}_{R R}
\end{array}\right]\left[\begin{array}{l}
\mathbf{q}_{L} \\
\mathbf{q}_{R}
\end{array}\right]=\left[\begin{array}{l}
\mathbf{f}_{L} \\
\mathbf{f}_{R}
\end{array}\right]
$$

The components in the above dynamic matrix can be rewritten as

$$
\begin{aligned}
& \mathbf{S}_{L L}=\mathbf{D}_{L L}-\mathbf{D}_{L I} \mathbf{D}_{I I}^{-1} \mathbf{D}_{I L} \\
& \mathbf{S}_{R R}=-\mathbf{D}_{R I} \mathbf{D}_{I I}^{-1} \mathbf{D}_{I R}+\mathbf{D}_{R R} \\
& \mathbf{S}_{L R}=-\mathbf{D}_{L I} \mathbf{D}_{I I}^{-1} \mathbf{D}_{I R}, \quad \mathbf{S}_{R L}=-\mathbf{D}_{R I} \mathbf{D}_{I I}^{-1} \mathbf{D}_{I L}
\end{aligned}
$$

Similarly, by employing the Bloch theorem, one characteristic quadratic equation can be derived as

$$
\left[\mathbf{S}_{R L}+\left(\mathbf{S}_{L L}+\mathbf{S}_{R R}\right) e^{i k \bar{l}}+\mathbf{S}_{L R} e^{2 i k \bar{l}}\right] \mathbf{q}_{L}=0
$$

where $\bar{l}=l_{1}+l_{2}$. This quadratic equation can be transformed into a linear characteristic problem by using the matrix transformation as follows:

$$
\mathbf{H}\left[\begin{array}{c}
\mathbf{q}_{L} \\
\mathbf{f}_{L}
\end{array}\right]=e^{i k \bar{l}}\left[\begin{array}{c}
\mathbf{q}_{L} \\
\mathbf{f}_{L}
\end{array}\right]
$$

where $\mathbf{H}$ is the transmission matrix obtained from the spectral element formulation, specified as

$$
\mathbf{H}=\left[\begin{array}{cc}
-\mathbf{S}_{L R}^{-1} \mathbf{S}_{L L} & -\mathbf{S}_{L R}^{-1} \\
\mathbf{S}_{R L}-\mathbf{S}_{R R} \mathbf{S}_{L R}^{-1} \mathbf{S}_{L L} & -\mathbf{S}_{R R} \mathbf{S}_{L R}^{-1}
\end{array}\right]
$$

Obviously, Eq. (40) can be reduced to an eigenproblem of $e^{i k \bar{l}}$ for a given $\omega$. The dispersive formulation can be expressed as

$$
\operatorname{det}\left[\mathbf{H}-e^{i k \bar{l}} \mathbf{I}\right]=0
$$

where $\mathbf{I}$ denotes the $4 \times 4$ unity matrix. At a given frequency $\omega$, the propagation constant $e^{i k \bar{l}}$ can be obtained by solving the eigenvalue problem by using the Cholesky decomposition of the eig functions in MATLAB software. This system has eight eigenvalues. Approximatively pure wavenumber for $k$ corresponds to the propagation mode, while other full complex eigenvalues that are not considered in this paper will decay exponentially in the propagation direction.

\section{TOPOLOGICAL TRANSITION}

This section employs the local resonance metastructure to realize the topological transition, where the dispersive behavior is analyzed via the finite element and spectral element methods. The base beam and central shim are made of aluminum and epoxy resin, respectively. PZT$5 \mathrm{H}$ is used as the piezo sheet. The end masses and connectors are steel, whose stiffnesses are much greater than those of the rest in the metastructure, regarded as rigid blocks. These material parameters are presented in Table. II.

The finite element model of the single-period cell is established via using the commercial finite element software COMSOL Multiphysics 5.4, as shown in the dashed box of Fig. 1(a). The Floquet-Bloch periodic conditions are applied at the left and right boundaries of this model, and the tetrahedral mesh is used to discrete the physical and geometrical domains. Thus, the capacitance ratio $\Phi$ can be defined as

$$
C_{n}=\Phi C_{p}^{s}
$$

where $C_{n}$ describes the negative capacitance value in the shunted circuit. Fig. 2(a) presents the band structure with $C_{n}=-1.4 C_{p}^{s}$, which can avoid the electrical instability ${ }^{44,45}$. The scattered dots and black lines give dispersive relationships by using the finite element and spectral element approaches, respectively. It is almost conformable to results obtained through numerical simulation and theoretical calculation. Indeed, the slight difference between the two types of results is also shown, especially in the high-frequency region. In spectral element formulation, the Euler-Bernoulli beam model has not considered the shearing displacement and inertia, while the numerical model is developed from the threedimensional solid. Besides, there is a bandgap between the two energy bands in the taken frequency range, shown in the purple region in Fig. 2(a), which is called the local resonant bandgap generated by the self-oscillation of the substructure.

In this system, we can tune the local resonant bandgap by adjusting the bimorph beam's dynamic stiffness so that the bandgap wavelength may be longer than the unit cell's length, also called sub-wavelength. As for the flexural wave, the effective wavelength $L$ in the sub-wavelength bandgap should satisfy the following expression $^{46}$

$$
L<\frac{c}{2 f}
$$

with

$$
c=\sqrt{\frac{h_{a} \omega}{2 \sqrt{3}} \sqrt{\frac{E_{b}}{\rho_{b}}}}
$$

where $c$ denotes the flexural wave's velocity in the base beam, $f$ is the actual frequency associated with the bandgap, $\omega$ describes the work angular frequency. After calculating the effective wavelength from Eq. (44) and Eq. (45), the active wavelength of the local resonates bandgap is longer than the unit cell's length. Hence, the 
TABLE II: The material parameters of the locally resonant metastructure ${ }^{39}$.

\begin{tabular}{lccccc}
\hline Property & Modulus & Density) & Dielectric \\
Unit & $\mathrm{GPa}$ & $103 \mathrm{~kg} / \mathrm{m}^{3}$ & $10^{-8} \mathrm{~F} / \mathrm{m}$ & $\begin{array}{c}\text { Piezoelectric } \\
10^{-10} \mathrm{C} / \mathrm{N}\end{array}$ & $\begin{array}{c}\text { Compliance } \\
\left.10^{-12} \mathrm{~m}^{2} / \mathrm{N}\right)\end{array}$ \\
\hline Epoxy & 4.1 & 1142 & - & - & - \\
PZT-5H & - & 7500 & 3.01 & 2.74 & 16.5 \\
Steel & 207 & 7780 & - & - & - \\
Aluminum & 70 & 2700 & - & - & - \\
\hline
\end{tabular}

(a)

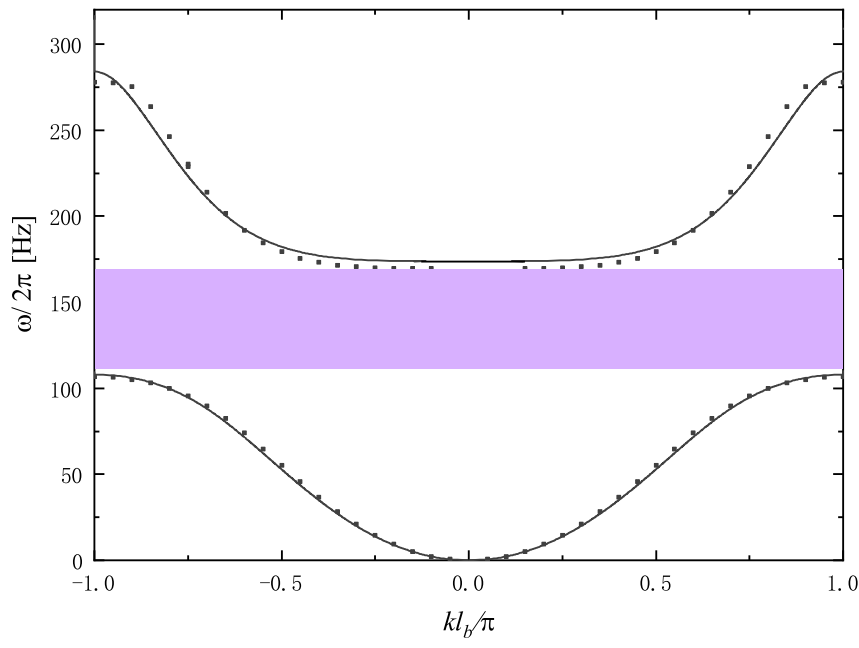

(b)

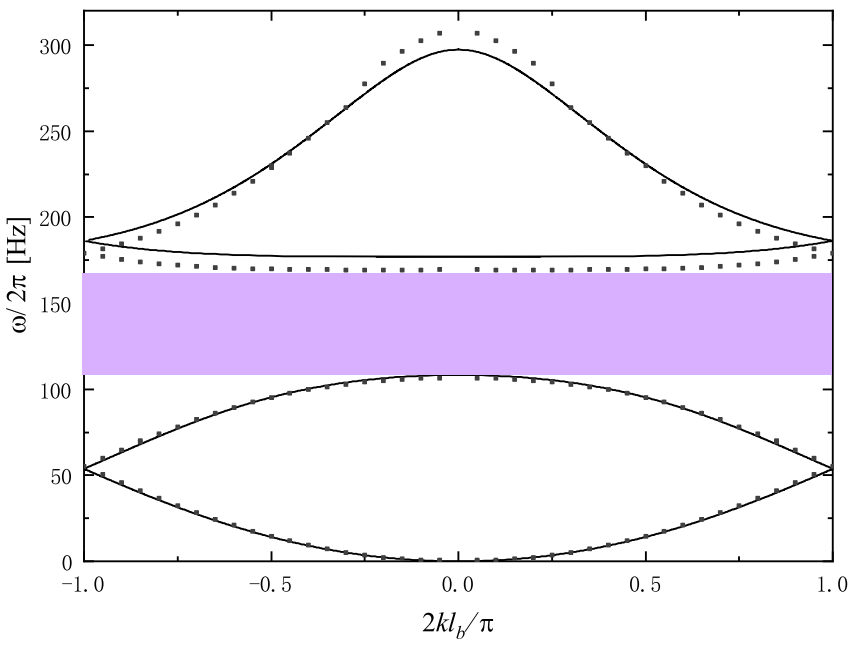

FIG. 2: The band structure of Fig. 1(a), where the black lines and red dots denote results obtained by using the spectral element method and finite element approach, respectively. (a) The single-period model; (b) The double-period model consisting of two primitive unit cells.

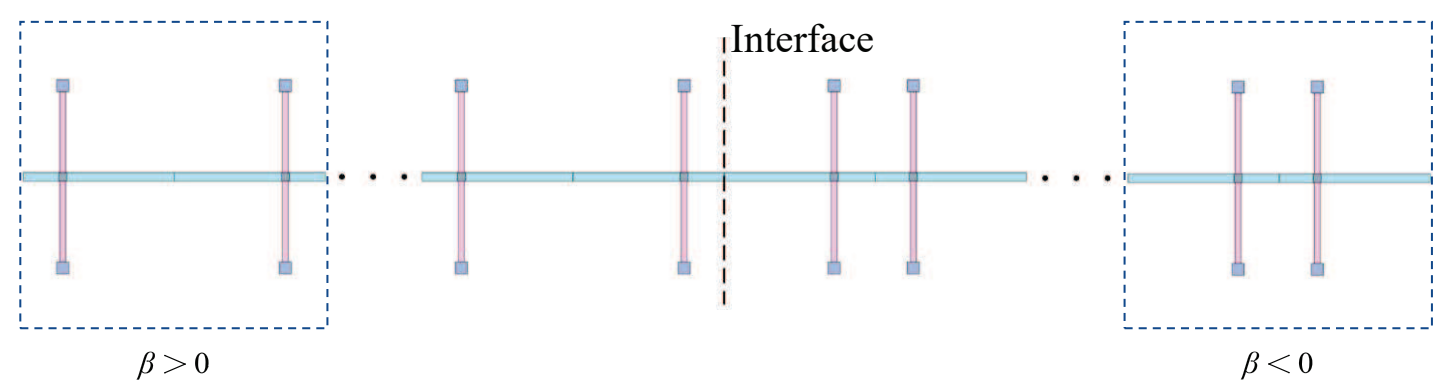

FIG. 3: The schematic map of the new metastructure obtained by adjusting the distance between the axes of two adjacent bimorph structures in the double-period model, which consists of two primitive unit cells. $l_{t}$ denotes the distance between the axes of two adjacent bimorph structures.

lower bandgap in the purple region has reached the subwavelength zone, as shown in Fig. 2(a).

We will merge two primitive cells into a new element to develop band-folding points at the Brillouin zone's high symmetric condition. These results obtained by employing the finite element and spectral element methods are shown in Fig. 2b. It can be seen that in the same frequency range, the double-periodic energy band has twice the lattice constant of the single-periodic model, and the Brillouin zone is reduced to half that of the singleperiodic model. The new model generates two Dirac points at $2 k l_{b}=\pi / 2$ as the lattice length of Fig. 2(b) doubles its original value of the primitive cell in Fig. 2(a). Thus, we also find that the bandgap generated by the local resonance keeps the same in Fig. 2(a) and Fig. 2(b). This local resonant bandgap is independent of the cell 

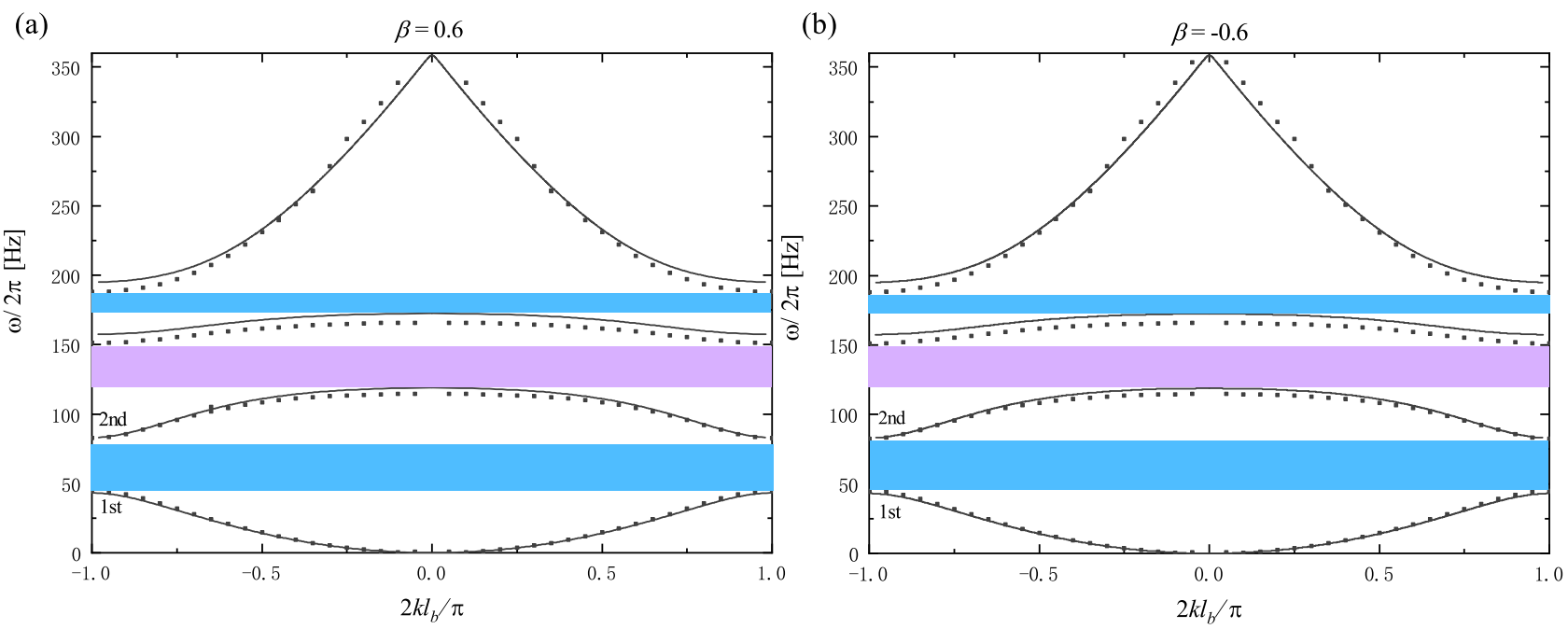

FIG. 4: The band structures of the new system in Fig. 3: (a) The dispersive relationship with $\beta=-0.6$, where the purple and blue regions denote the local resonance and Bragg bandgaps, respectively; (b) The dispersive relationship with $\beta=0.6$. The black lines and dots denote results obtained by using the spectral element method and finite element approach, respectively.

TABLE III: The mode shapes at $2 k l_{b}=0$ and $2 k l_{b}=\pi$ of the first and second bands with $\beta=-0.6$ and $\beta=0.6$.

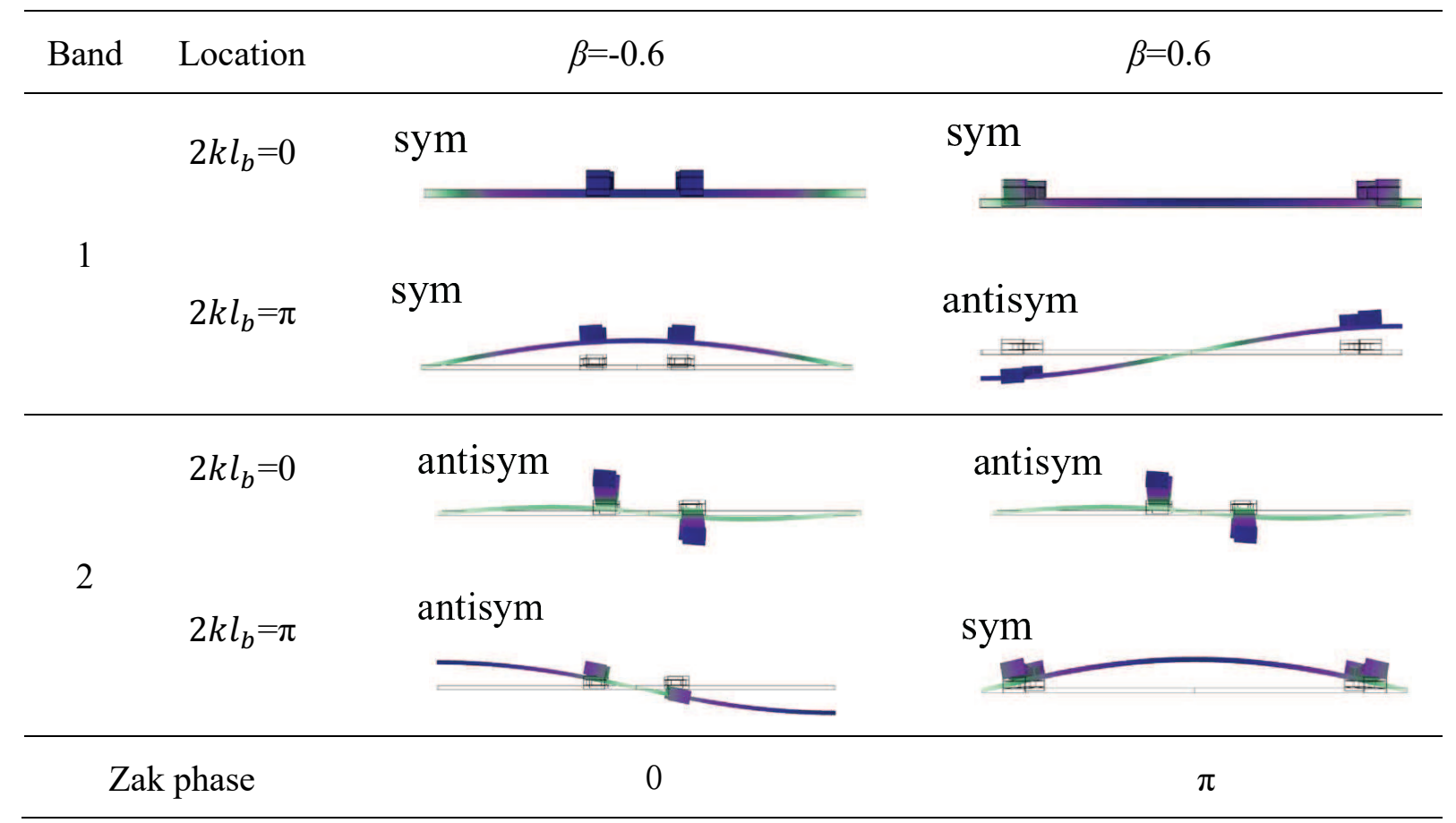

length in the metastructure.

In the double-period model, $l_{b}$ denotes the distance between the axes of two adjacent bimorph structures in Fig. $1(\mathrm{a})$. Note that the lattice length becomes $2 l_{b}$ in the new system. Naturally, the distance between the axes of two adjacent bimorph structures can be utilized to break the spatial symmetric to unfold two Dirac points of Fig. 2(b), which further develops two Bragg bandgaps, as shown in Fig. 3. The dimensionless parameter $\beta$ associated with the distance between the axes of two adjacent bimorph 
(a)

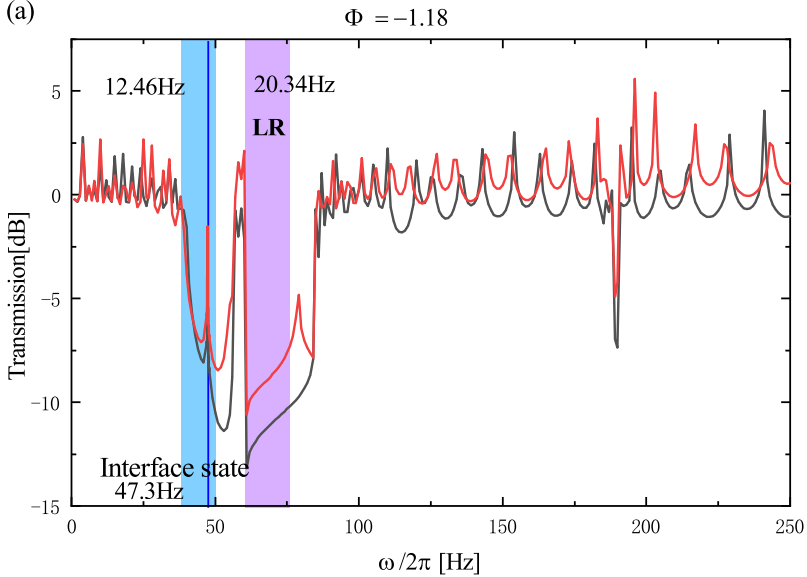

(c)

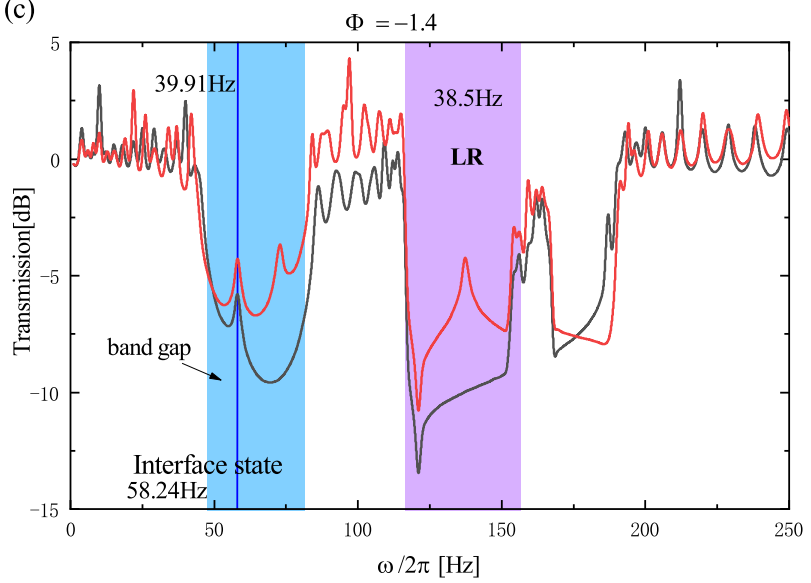

(b)

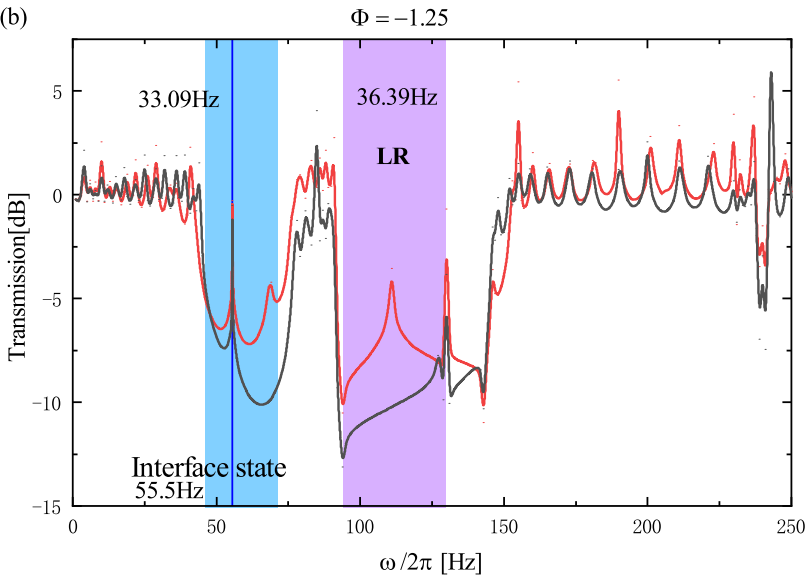

(d)

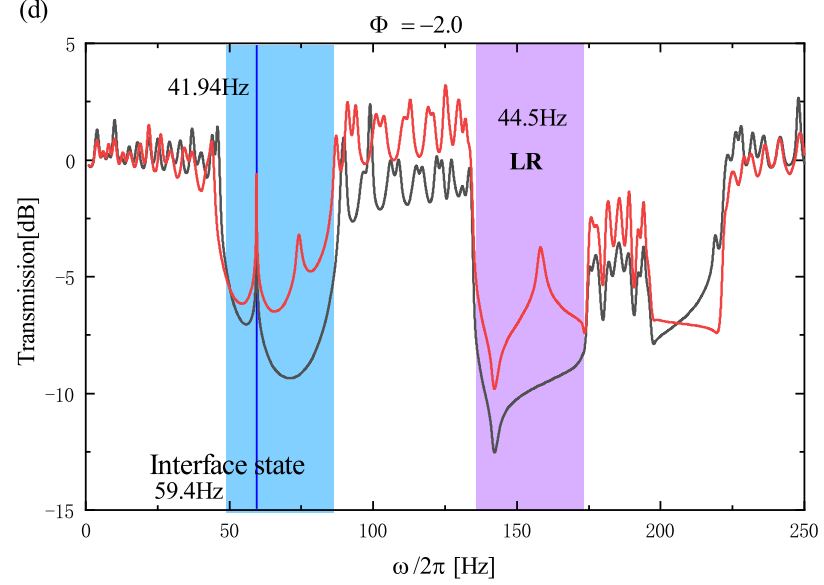

FIG. 5: The frequency response functions are calculated in the heterostructure with different capacitance ratios, where the blue and purple regions denote the local resonance and Bragg bandgaps, respectively. There are one localized mode in the lower Bragg scattering bandgap. (a) $\Phi=-1.18$; (b) $\Phi=-1.25$; (c) $\Phi=-1.4$; (d) $\Phi=-2.0$.

The red and blue lines are transmitted from the right and left ends, respectively.

beam in Fig. 3 is defined as

$$
\beta=\frac{l_{t}-l_{b}}{l_{b}}
$$

where $l_{t}$ describes the distance between the axes of two adjacent bimorph structures in the new system of Fig. 3. Obviously, Fig. 2(b) gives the band structures with $\beta=1$. When $\beta \neq 1$, the translational symmetry in the infinitely periodic structure is broken to open the folding point to form two new band gaps. Fig. 4(a) and Fig. 4(b) plot the dispersive relationships with $\beta=0.6$ and $\beta=-0.6$, respectively. We can find that the bandgap of the purple region in Fig. 4 remains the same as that of Fig. 2. Hence, the local resonate bandgap in Fig. 4 is irrelevant to $\beta$ and still stays in the sub-wavelength zone. Besides, Fig. 4(a) and Fig. 4(b) have a common new bandgap of the blue region, which is developed between the $1^{\text {st }}$ and $2^{\text {nd }}$ bands. The active bandgap generated by the band-folding ${ }^{47}$ method can be realized in the subwavelength zone.

It is necessary to analyze the topological properties of energy band structures in Fig. 4(a) and Fig. 4(b), to realize the sub-wavelength interface state. In the onedimensional periodic structure, the Zak phase can be regarded as one topological invariant ${ }^{48}$. The Zak phase is a special Berry phase with the expression

$$
\theta_{m}^{Z a k}=i \iint_{\Omega} U^{*}(k) \frac{\partial U(k)}{\partial k} d V d k
$$

where $\theta_{m}^{Z a k}$ is the Zak phase of the $\mathrm{m}^{\text {th }}$ energy band. $U(k)$ describes the mode shape of the $\mathrm{m}^{\text {th }}$ energy band in the cell. The superscript $*$ denotes the conjugate, and $\Omega$ is the volume of the cell.

It is complex to calculate the Zak phase directly in the continuum model. Another qualitative approach associated with the Zak phase is employed to derive the energy band topological properties in this paper. The Zak phase $\theta_{m}^{Z a k}$ of each band is connected with the parities of the eigenmodes as follows ${ }^{49,50}$ :

$$
\frac{\theta_{m}^{Z a k}}{\pi}=\frac{1}{2}\left[\eta\left(2 k l_{b}=0\right)-\eta\left(2 k l_{b}=\pi\right)\right]: \bmod (2)
$$


(a)

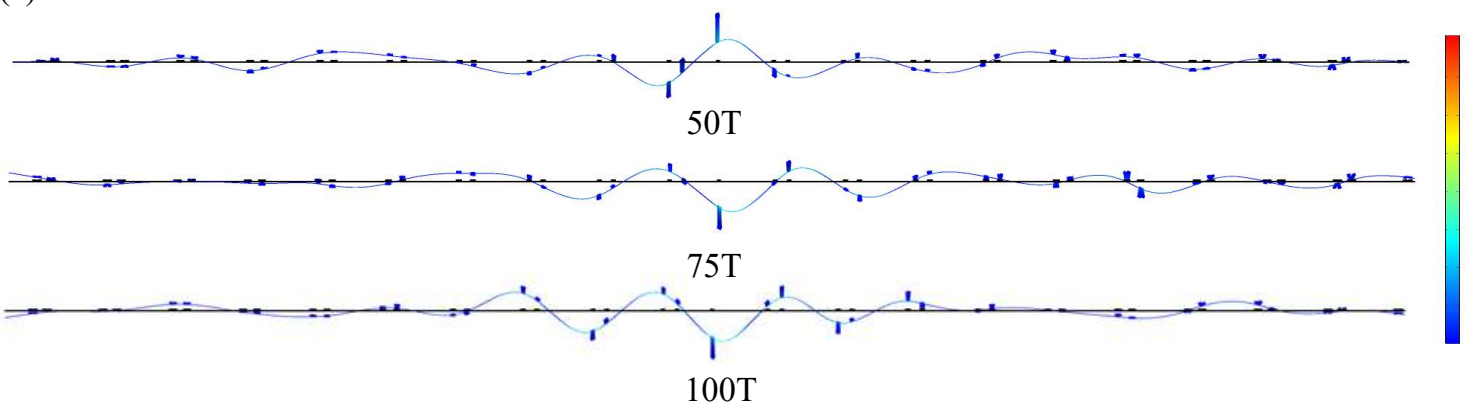

(b) Pass band $(20 \mathrm{~Hz})$

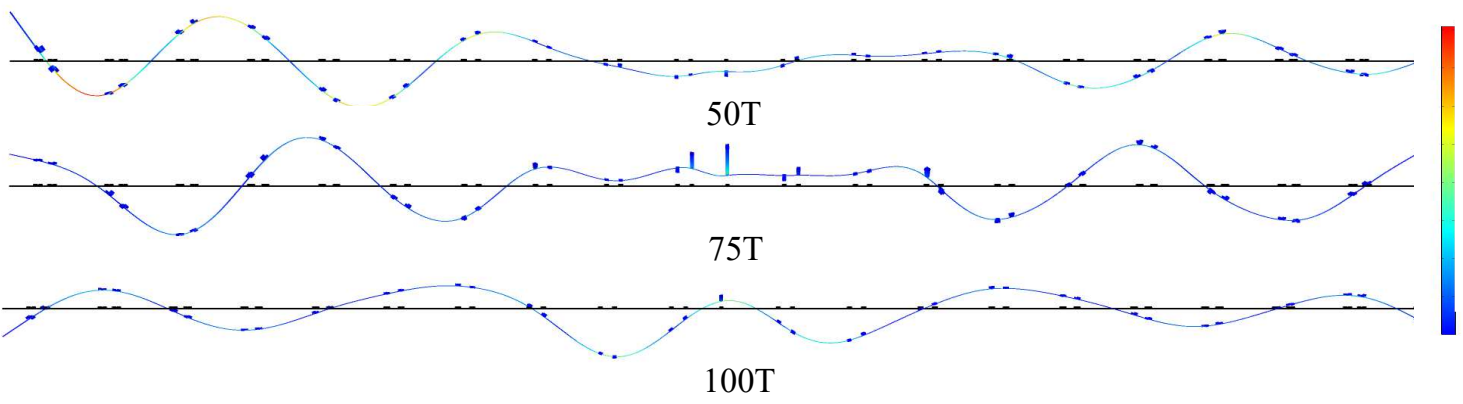

(c)

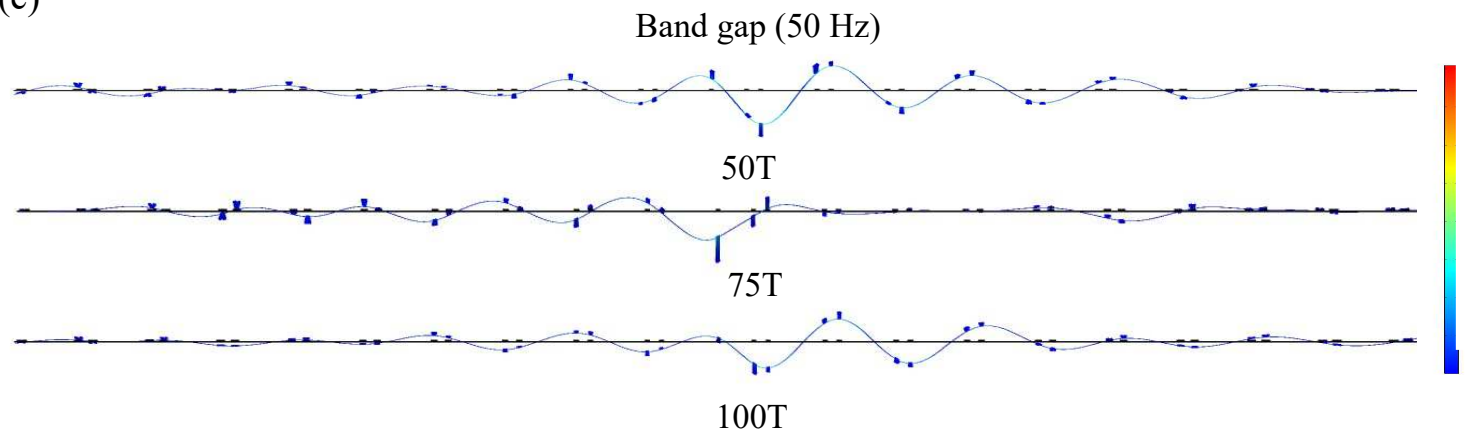

FIG. 6: The flexural displacement field of the $\Phi=-1.4$ heterostructure with three kinds of frequency excitation over $100 \mathrm{~T}$. (a) When $\omega / 2 \pi=58.24 \mathrm{~Hz}$ for an interface mode, the energy is mainly concentrated at the interface; (b) When $\omega / 2 \pi=20 \mathrm{~Hz}$, the energy is normally transmitted to both ends; (c) When $\omega / 2 \pi=50 \mathrm{~Hz}$, the energy is also concentrated at the interface. However, this degree of concentration is much smaller than that of the first term.

where $\eta$ is the parity at different symmetry points. The symmetry mode shape is defined to have an odd parity -, while the antisymmetry structure has an even parity + . The qualitative approach analyzes the mode shapes at $2 k l_{b}=0$ and $2 k l_{b}=\pi$ in the energy band to predict the topological properties. As shown in Table. III, the mode shapes at $2 k l_{b}=0$ and $2 k l_{b}=\pi$ of the first and second energy bands in $\beta=-0.6$ are both positive symmetric or antisymmetric. We obtain that the Zak phases of the first and second bands become zero at $\beta=-0.6$. However, only one of two eigenmodes at $2 k l_{b}=0$ and $2 k l_{b}=\pi$ of the first or second bands turns symmetry or antisymmetry, so that their Zak phases both are $\pi$ at $\beta=0.6$.

In addition, the topological behavior of the bandgap can also be verified by the corresponding impedance sign. If the bandgaps possess different topological properties, the corresponding impedance values will present the opposite case. The impedance sign is defined as

$$
\operatorname{sgn}\left(\zeta^{n}\right)=(-1)^{n}(-1)^{l} \exp \left(i \sum_{m=0}^{n-1} \theta_{m}^{Z a k}\right)
$$

where $\operatorname{sgn}\left(\zeta^{n}\right)$ describes the impedance sign of the $n^{t h}$ bandgap. $l$ is the number of the bands. From Eq. (49), the impedance signs of the first and second bandgaps become positive in $\beta>0$. When $\beta<0, \operatorname{sgn}\left(\zeta^{n}\right)$ of the first bandgaps turn into a negative region, but the second bandgap continues to maintain a positive sign. It is also noted that that the Bragg bandgap's topological phase 
transition occurs following a shift in the variation of $\beta$. Nevertheless, the second bandgap's topological behavior remains unchanged. It is not easy to achieve the topological interface state in the local resonance bandgap. Besides, the lower bandgaps associated with $\beta=0.6$ and $\beta=-0.6$ have a common region, while the first bandgap in Fig. 4(a) performs the direct opposite of Fig. 4(b). Therefore, the band-folding mechanism can be employed to realize the subwavelength interface state associated with the Bragg bandgap in this metastructure.

\section{TUNABLE TOPOLOGICAL INTERFACE STATE}

In this section, our previous predictions can be firstly confirmed by numerically computing the forced harmonic response. The lower frequency bandgaps with $\beta=-0.6$ and $\beta=0.6$ have one common region while performing different topological properties. This results in a topological interface state within this bandgap demonstrated in the frequency response. Thereupon, one heterostructure with ten unit cells on each side of the interface is constructed, as shown in Fig. 3. Thus, the frequency response function can be defined as

$$
T_{1}=\log _{10}\left|\frac{u_{\text {end }}}{u_{\text {in }}}\right|
$$

where $u_{\text {in }}$ denotes the input displacement at the exciting end. $u_{\text {end }}$ describes the output displacement at the other end. This frequency response is normalized to the excitation amplitude. The left and right ends of the structure are excited separately under the shunt circuit with negative capacitance $C_{n}=-1.4 C_{p}^{s}$, and their frequency response curves are shown in Fig. 5(c) by employing the finite element method, where the red and blue lines are transmitted from the right and left end, respectively. The blue and purple areas represent the Bragg scattering and local resonate bandgaps, and they match exactly with the analytical results of Fig. 4. It is found that the transmission peaks associated with the left and right excited ends both stay the same value in the bandgap region. Thus, the second peak of the red line in the bandgap indicates the non-trivial topological behavior. Therefore, this heterostructure with two topological properties supports one localized mode at the interface frequency $(58.24 \mathrm{~Hz})$ within the lower frequency bandgap. These finds also confirm our predictions about topological interface modes in Section 4.

The transient response is used to illustrate the dynamic behavior of the interface mode. We compute the frequency response over $100 \mathrm{~T}(T=2 \pi / \omega)$ by imposing a displacement $u_{i n}=1 \times 10^{-4} \sin (2 \pi \omega t)$ at the interface in the finite element model. The velocity fields with different time instances are obtained by using the finite element method. Fig. 6 gives the flexural displacement field with three types of frequency excitation: (a) $58.24 \mathrm{~Hz}$; (b) $20 \mathrm{~Hz}$; (c) $50 \mathrm{~Hz}$. In this system, the first values yield

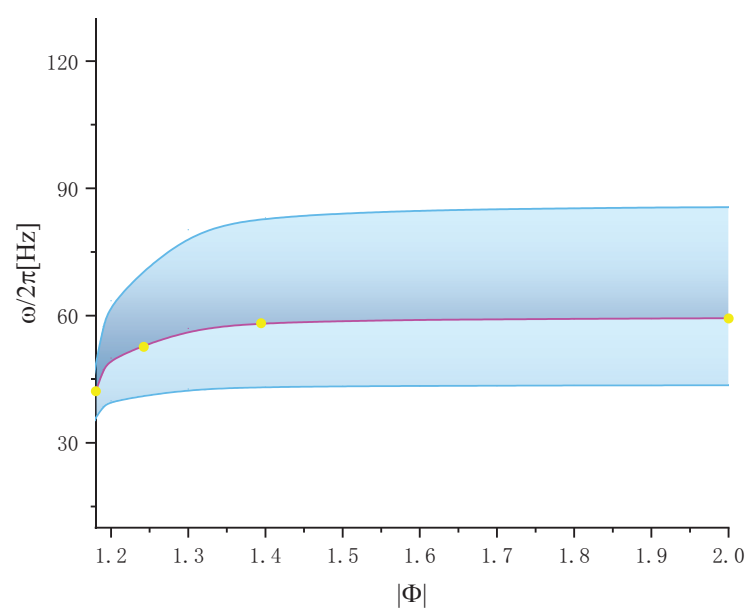

FIG. 7: The relationships between the capacitance ratio and bandgap characteristics, including the topological properties, widths, and interface frequencies.

the interface frequency generated by the Bragg scattering bandgap; The second item is situated at passbands of Fig. 4(a) and Fig. 4(b); The final value shows one frequency located in the lower frequency bandgap, but is not the interface frequency. It is easily discovered that the magnitude associated with the localized mode reaches a maximum near the interface and then reduces sharply away from the interface in Fig. 6(a). Besides, these transient velocity fields denote the typical field distribution of the interface state. The velocity field is highly concentrated at the interface and never passes to both the left and right ends. At $\omega / 2 \pi=20 \mathrm{~Hz}$, the velocity field is typically transmitted to both ends of the heterostructure, as shown in Fig. 6(b). Moreover, Fig. 6(c) gives results of $50 \mathrm{~Hz}$ at the common bandgap. This energy is also concentrated at the interface, and is barely transmitted to the left and right ends. However, it is found that the degree of concentration is smaller than that of the interface state. By comparison, the energy is highly concentrated at the interface when the excitation frequency equals the localized frequency concerning the topological interface state.

In this metastructure, the shunting circuit is employed to tune the effective stiffness associated with the bimorph beam to control the local resonate bandgap and corresponding interface frequency. Fig. 7 plots the relationship between the capacitance parameter $\Phi$ and lower frequency bandgap when the geometrical and material parameters keep the same as those of Fig. 3. The bandgap width in the lower frequency region increases as the absolute value $|\Phi|$ increases. The frequency response curves with four different $\Phi$ are utilized to confirm the trend of $|\Phi|$ with respect to the width of the lower frequency bandgap, as shown in Fig. 5. Thus, this type of heterostructure yields one topological interface state in the lower frequency region during the shunting circuit changing. In order to show the trend of the interface fre- 


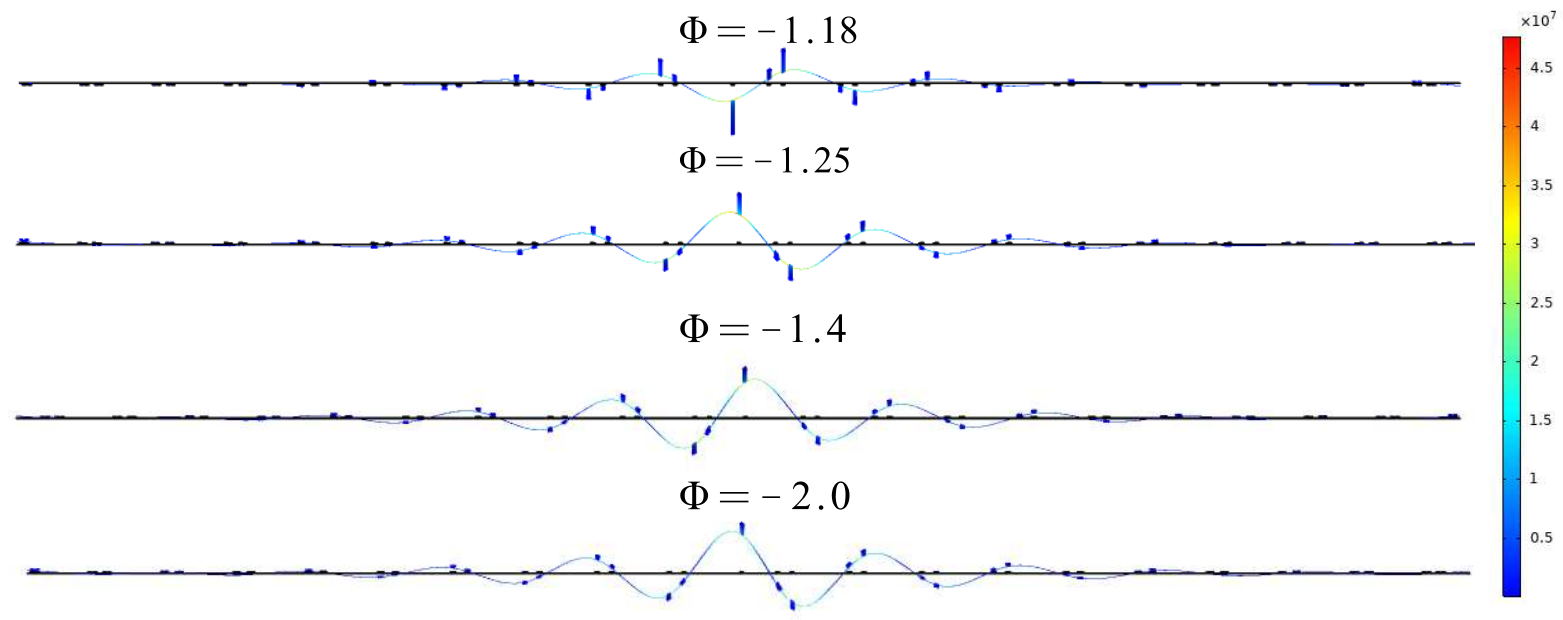

FIG. 8: The mode shapes of the interface modes with four different capacitance ratios.

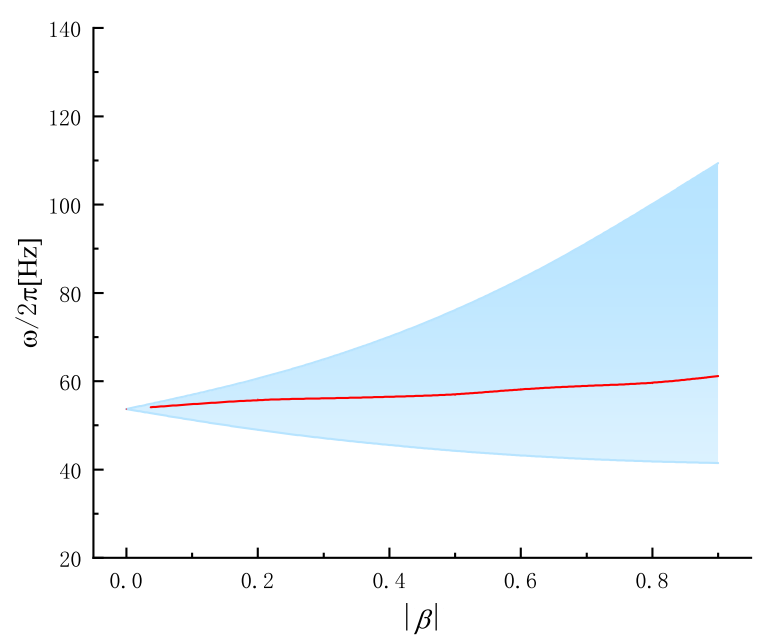

FIG. 9: The relationship between $\beta$ and bandgap characteristics, including the topological properties, widths, and interface frequencies.

quency, the response curves with the four capacitance ratios -1.18 (Fig. 5(a)), -1.25 (Fig. 5(b)), -1.4(Fig. 5(c)) and $-2.0($ Fig. $5(\mathrm{~d}))$ are calculated by using the finite element method, whose transmission peak associated with the specific interface frequencies are located at $20 \mathrm{~Hz}$, $55.5 \mathrm{~Hz}, 58.24 \mathrm{~Hz}$ and $59.4 \mathrm{~Hz}$, respectively. The frequency of the local resonant bandgap increases when $|\Phi|$ becomes larger to enhance the effective stiffness of the bimorph beam, which further raises the topological interface frequency in the metastructure. Besides, Fig. 8 shows the mode shapes of the topological interface state with corresponding four different $|\Phi|$. We can find that wave localization at the interface changes significantly higher from Fig. 8 when the lower frequency bandgap becomes wider with the increase of $|\Phi|$.

Besides, the effect of $\beta$ on bandgap characteristics is analyzed by using the frequency response technique, as shown in Fig. 9 . The $\beta$ values of the left and right lattices show one sign reversal, similar to Fig. 3. Clearly, the interface state disappears by closing the Dirac point at $\beta=1$. As the absolute value of $\beta$ becomes small, the breaking degree of the symmetric turns more apparent. Hence, the bandgap and interface frequency increase with a decrease of $\beta$. This design may be extended to other two-dimensional and three-dimensional metastructure systems whereby tunable elastic topological insulators are strategically employed to finish wave localization and directional propagation, with potential application to acoustic devices, non-destructive testing, and energy harvest in particular alterable condition.

\section{CONCLUSION}

We analyze the tunable low-frequency interface state of flexural wave in the locally resonant metastructure consisting of beam-like local resonances and shunting piezoelectric circuits. Inspired by the zone-folding in frequency band structures, doubling the primitive unit cell develops the Dirac point in the high symmetry boundary. Thus, the beam-like local resonator is utilized to produce the sub-wavelength bandgap by the external electrical circuit so that this metastructure can obtain the low-frequency folding point. The manipulation of the distance between two adjacent resonances is applied to break the mirrorsymmetric to open this band-folding point to develop one new Bragg scattering bandgap in the sub-wavelength region. Moreover, the band inversion and topological phase transition have been demonstrated during the distance variation between two adjacent resonances.

The new heterostructure is constructed in this paper, composed of two sub-structures with different impedance signs. The interface state is discovered and confirmed in the frequency response of the heterostructure. We 
also analyze the propagation behavior on wave localized states in the sub-wavelength region employing the steady and transient analysis. It is found that the velocity field is highly concentrated at the interface when an interface frequency is excited. These energies are less to be transferred to both ends. Besides, the relationship between the interface frequency and capacitance ratio is shown. We discover that the interface frequency increases with an increase in the capacitance ratio. Besides, the influence of the distance parameter on the topological interface mode is also discussed.

\section{ACKNOWLEDGMENTS}

The authors wish to acknowledge the support from the National Natural Science Foundation of China (Grant No. 12002094 and 11972160), the Natural Science Foundation of Guangdong Province (Grant No. 2018A030310310), and Guangxi Youth Innovative Talents research project (Grant No. 20200312)

\section{DATA AVAILABILITY}

The data that support the results of this paper are available from the corresponding author upon reasonable request.

\section{REFERENCES}

${ }^{1}$ Z. Yang, F. Gao, X. Shi, X. Lin, Z. Gao, Y. Chong, and B. Zhang, "Topological acoustics," Physical Review Letters 114, 114301 (2015).

${ }^{2}$ M. Xiao, G. Ma, Z. Yang, P. Sheng, Z. Q. Zhang, and C. T. Chan, "Geometric phase and band inversion in periodic acoustic systems," Nature Physics 11, 240-244 (2015).

${ }^{3}$ S. H. Mousavi, A. B. Khanikaev, and Z. Wang, "Topologically protected elastic waves in phononic metamaterials," Nature Communications 6, 8682 (2015).

${ }^{4}$ H. Chen, H. Nassar, A. N. Norris, G. K. Hu, and G. L. Huang, "Elastic quantum spin hall effect in kagome lattices," Physical Review B 98 (2018).

${ }^{5}$ R. Chaunsali, C.-W. Chen, and J. Yang, "Subwavelength and directional control of flexural waves in zone-folding induced topological plates," Physical Review B 97 (2018).

${ }^{6}$ T.-W. Liu and F. Semperlotti, "Tunable acoustic valley-hall edge states in reconfigurable phononic elastic waveguides," Physical Review Applied 9 (2018).

${ }^{7}$ J. Lu, C. Qiu, W. Deng, X. Huang, F. Li, F. Zhang, S. Chen, and Z. Liu, "Valley topological phases in bilayer sonic crystals," Physical Review Letters 120, 116802 (2018).

${ }^{8}$ M. Miniaci, R. K. Pal, B. Morvan, and M. Ruzzene, "Experimental observation of topologically protected helical edge modes in patterned elastic plates," Phys. Rev. X 8, 031074 (2018).

${ }^{9}$ S. Li, D. Zhao, H. Niu, X. Zhu, and J. Zang, "Observation of elastic topological states in soft materials," Nature Communications 9, 1370 (2018).

${ }^{10}$ M. Miniaci, R. Pal, B. Morvan, and M. Ruzzene, "Experimental observation of topologically protected helical edge modes in patterned elastic plates," Physical Review X 8, 031074 (2018).
${ }^{11}$ Muhammad, W. Zhou, and C. W. Lim, "Topological edge modeling and localization of protected interface modes in $1 \mathrm{~d}$ phononic crystals for longitudinal and bending elastic waves," International Journal of Mechanical Sciences 159, 359-372 (2019).

${ }^{12}$ X. Zhang, M. Xiao, Y. Cheng, M.-H. Lu, and J. Christensen, "Topological sound," Communications Physics 1 (2018).

${ }^{13}$ C. He, X. Ni, H. Ge, X.-C. Sun, Y.-B. Chen, M.-H. Lu, X.-P. Liu, and Y.-F. Chen, "Acoustic topological insulator and robust one-way sound transport," Nature Physics 12, 1124-1129 (2016).

${ }^{14}$ H. Liu, H. Xia, M. Zhuang, Z. Long, C. Liu, J. Cui, B. Xu, Q. Hu, and Q. H. Liu, "Reverse time migration of acoustic waves for imaging based defects detection for concrete and cfst structures," Mechanical Systems and Signal Processing 117, 210-220 (2019).

${ }^{15}$ H. Liu, Y. Qi, Z. Chen, H. Tong, C. Liu, and M. Zhuang, "Ultrasonic inspection of grouted splice sleeves in precast concrete structures using elastic reverse time migration method," Mechanical Systems and Signal Processing 148, 107152 (2021).

${ }^{16}$ R. Fleury, D. L. Sounas, C. F. Sieck, M. R. Haberman, and A. Alu, "Sound isolation and giant linear nonreciprocity in a compact acoustic circulator," Science 343, 516-519 (2014).

${ }^{17}$ R. Ssstrunk and S. D. Huber, "Observation of phononic helical edge states in a mechanical topological insulator," Science $\mathbf{3 4 9}$, 47-50 (2015).

${ }^{18}$ P. Wang, L. Lu, and K. Bertoldi, "Topological phononic crystals with one way elastic edge waves," Physical Review Letters 115, 104302 (2015).

${ }^{19}$ W. Zhou, Y. Su, Muhammad, W. Chen, and C. Lim, "Voltagecontrolled quantum valley hall effect in dielectric membranetype acoustic metamaterials," International Journal of Mechanical Sciences 172, 105368 (2020).

${ }^{20}$ A. Darabi, M. Collet, and M. J. Leamy, "Experimental realization of a reconfigurable electroacoustic topological insulator," Proc Natl Acad Sci U S A 117, 16138-16142 (2020).

${ }^{21}$ Q. Zhang, Y. Chen, K. Zhang, and G. Hu, "Dirac degeneracy and elastic topological valley modes induced by local resonant states," Physical Review B 101 (2020).

${ }^{22}$ Z. Chen, W. Zhou, and C. Lim, "Tunable frequency response of topologically protected interface modes for membrane-type metamaterials via voltage control," Journal of Sound and Vibration 494, 115870 (2021).

${ }^{23}$ Y. Si-Yuan, H. Cheng, W. Zhen, L. Fu-Kang, S. Xiao-Chen, L. Zheng, L. Hai-Zhou, L. Ming-Hui, L. Xiao-Ping, and C. YanFeng, "Elastic pseudospin transport for integratable topological phononic circuits," Nature Communications 9, 3072 (2018).

${ }^{24}$ H. Wang, D. Liu, W. Fang, S. Lin, Y. Liu, and Y. Liang, "Tunable topological interface states in one-dimensional extended granular crystals," International Journal of Mechanical Sciences 176, 105549 (2020).

${ }^{25}$ Y. Liu, L. Jin, H. Wang, D. Liu, and Y. Liang, "Topological interface states in translational metamaterials for sub-wavelength in-plane waves," International Journal of Mechanical Sciences 197, 106308 (2021).

${ }^{26}$ Y. Huang, Y. Huang, W. Chen, and R. Bao, "Flexible manipulation of topologically protected waves in one-dimensional soft periodic plates," International Journal of Mechanical Sciences 170, 105348 (2020).

${ }^{27}$ H. Tiersten and B. Sinha, "Temperature dependence of the resonant frequency of electroded doubly-rotated quartz thicknessmode resonators," Journal of Applied Physics 50, 8038-8051 (1979).

${ }^{28}$ Z. Chen, G. Wang, W. Zhou, and C. Lim, "Elastic foundation induced wide bandgaps for actively-tuned topologically protected wave propagation in phononic crystal beams," International Journal of Mechanical Sciences 194, 106215 (2021).

${ }^{29}$ X. Chen, X. Xu, S. Ai, H. Chen, Y. Pei, and X. Zhou, "Active acoustic metamaterials with tunable effective mass density by gradient magnetic fields," Applied Physics Letters 105, 071913 (2014).

${ }^{30} \mathrm{~K}$. Yi and M. Collet, "Broadening low-frequency bandgaps in locally resonant piezoelectric metamaterials by negative capaci- 
tance," Journal of Sound and Vibration 493, 115837 (2021).

${ }^{31}$ L. Airoldi and M. Ruzzene, "Design of tunable acoustic metamaterials through periodic arrays of resonant shunted piezos," New Journal of Physics 13, 113010 (2011).

${ }^{32}$ O. Thorp, M. Ruzzene, and A. Baz, "Attenuation of wave propagation in fluid-loaded shells with periodic shunted piezoelectric rings," Smart Materials and Structures 14, 594-604 (2005).

${ }^{33}$ A. Spadoni, M. Ruzzene, and K. Cunefare, "Vibration and wave propagation control of plates with periodic arrays of shunted piezoelectric patches," Journal of Intelligent Material Systems and Structures 20, 979-990 (2009).

${ }^{34}$ F. Casadei, M. Ruzzene, L. Dozio, and K. A. Cunefare, "Broadband vibration control through periodic arrays of resonant shunts: experimental investigation on plates," Smart Materials and Structures 19, 015002 (2009).

${ }^{35}$ W. Zhou, B. Wu, Z. Chen, W. Chen, C. Lim, and J. Reddy, "Actively controllable topological phase transition in homogeneous piezoelectric rod system," Journal of the Mechanics and Physics of Solids 137, 103824 (2020).

${ }^{36}$ W. Zhou, W. Chen, M. Destrade, and C. Lim, "Actively controllable topological phase transition in phononic beam systems," International Journal of Mechanical Sciences 180, 105668 (2020).

${ }^{37}$ Y. Xiao, J. Wen, D. Yu, and X. Wen, "Flexural wave propagation in beams with periodically attached vibration absorbers: bandgap behavior and band formation mechanisms," Journal of Sound and Vibration 332, 867-893 (2013).

${ }^{38}$ S. El-Borgi, R. Fernandes, P. Rajendran, R. Yazbeck, J. Boyd, and D. Lagoudas, "Multiple bandgap formation in a locally resonant linear metamaterial beam: Theory and experiments," Journal of Sound and Vibration 488, 115647 (2020).

${ }^{39}$ W. Zhou, W. Chen, Z. Chen, and C. W. Lim, "Actively controllable flexural wave band gaps in beam-type acoustic metamaterials with shunted piezoelectric patches," European Journal of Mechanics-A/Solids 77, 103807 (2019).

${ }^{40}$ Y. Liu, S. Lin, Y. Li, C. Li, and Y. Liang, "Numerical investigation of rayleigh waves in layered composite piezoelectric structures using the siga-pml approach," Composites Part B: Engineering 158, 230-238 (2019).
${ }^{41}$ Y. Liang, Y. Li, Y. Liu, Q. Han, and D. Liu, "Investigation of wave propagation in piezoelectric helical waveguides with the spectral finite element method," Composites Part B: Engineering 160, 205-216 (2019).

${ }^{42}$ G. Hu, A. C. M. Austin, V. Sorokin, and L. Tang, "Metamaterial beam with graded local resonators for broadband vibration suppression," Mechanical Systems and Signal Processing 146, $106982(2021)$.

${ }^{43}$ Y. Xiao, J. Wen, D. Yu, and X. Wen, "Flexural wave propagation in beams with periodically attached vibration absorbers: Bandgap behavior and band formation mechanisms," Journal of Sound and Vibration 332, 867-893 (2013).

${ }^{44}$ A. R. M. Siddique, S. Mahmud, and B. V. Heyst, "A comprehensive review on vibration based micro power generators using electromagnetic and piezoelectric transducer mechanisms," Energy Conversion and Management 106, 728-747 (2015).

${ }^{45}$ J. Gripp and D. Rade, "Vibration and noise control using shunted piezoelectric transducers: A review," Mechanical Systems and Signal Processing 112, 359-383 (2018).

${ }^{46}$ G. Wang, J. Cheng, J. Chen, and Y. He, "Multi-resonant piezoelectric shunting induced by digital controllers for subwavelength elastic wave attenuation in smart metamaterial," Smart Materials and Structures 26, 025031 (2017).

${ }^{47}$ L. Fan, Y. He, X. Zhao, and X.-a. Chen, "Subwavelength and broadband tunable topological interface state for flexural wave in one-dimensional locally resonant phononic crystal," Journal of Applied Physics 127, 235106 (2020).

${ }^{48}$ M. Xiao, G. Ma, Z. Yang, P. Sheng, Z. Q. Zhang, and C. T. Chan, "Geometric phase and band inversion in periodic acoustic systems," Nature Physics 11, 240-244 (2015).

${ }^{49}$ Z. Zhang, Y. Cheng, X. Liu, and J. Christensen, "Subwavelength multiple topological interface states in one-dimensional labyrinthine acoustic metamaterials," Physical Review B 99 (2019).

${ }^{50}$ H.-X. Wang, G.-Y. Guo, and J.-H. Jiang, "Band topology in classical waves: Wilson-loop approach to topological numbers and fragile topology," New Journal of Physics 21 (2019). 
(a)
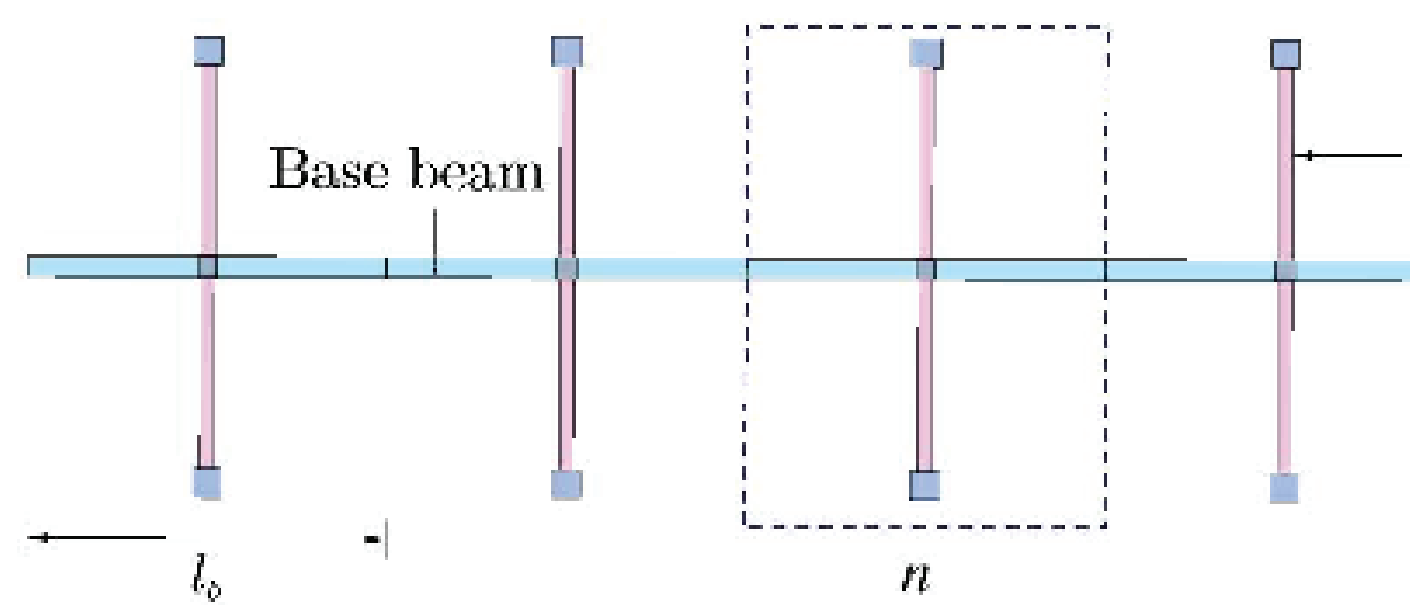

$2 l_{s}$

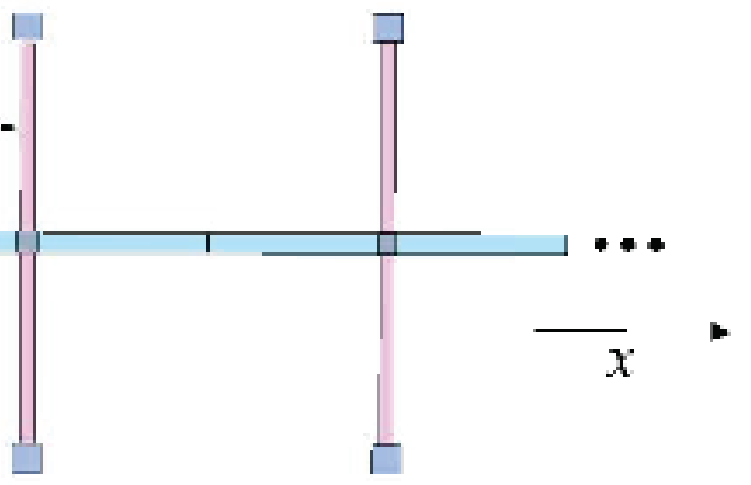

(b)

\ $2 h_{p}+h_{s}-h_{c}$

Connector

Mass block

(c)

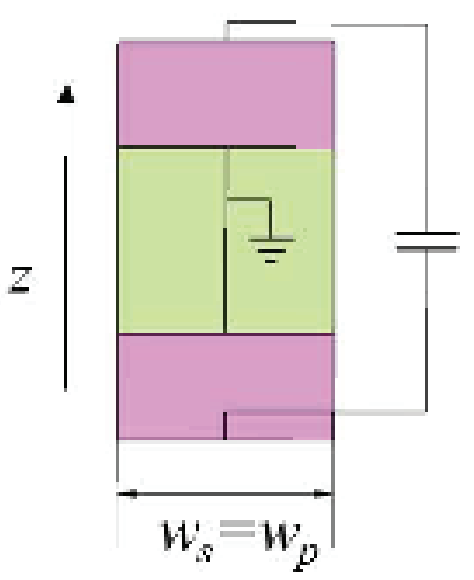




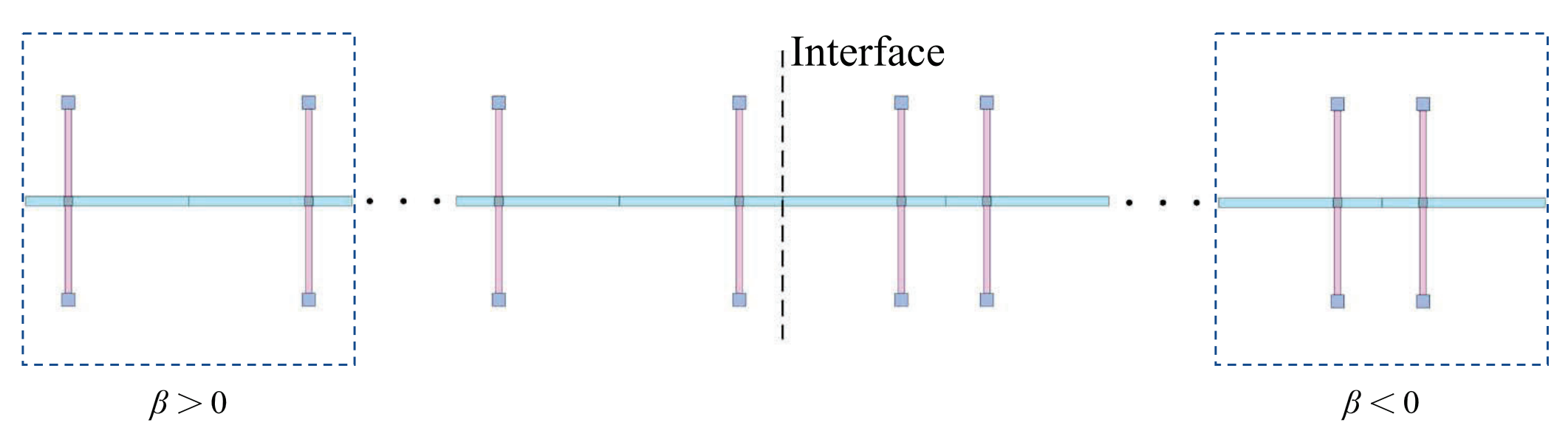


(a)

$\Phi=-1.18$

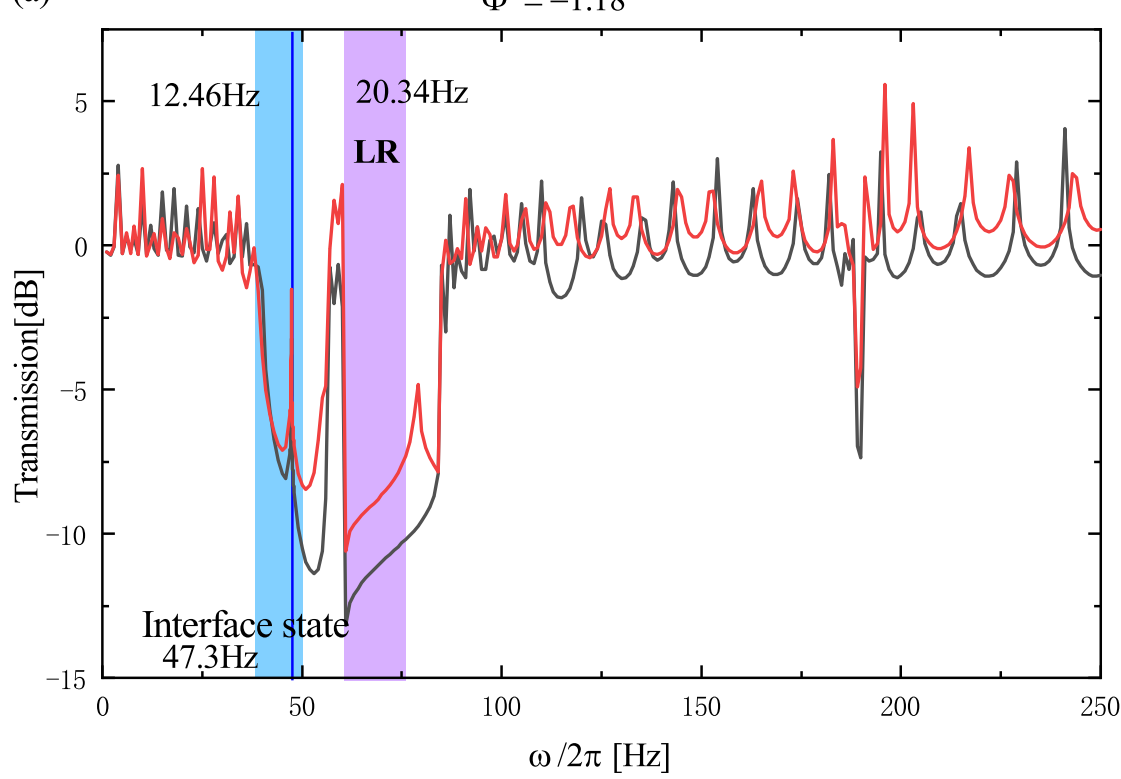

(c)

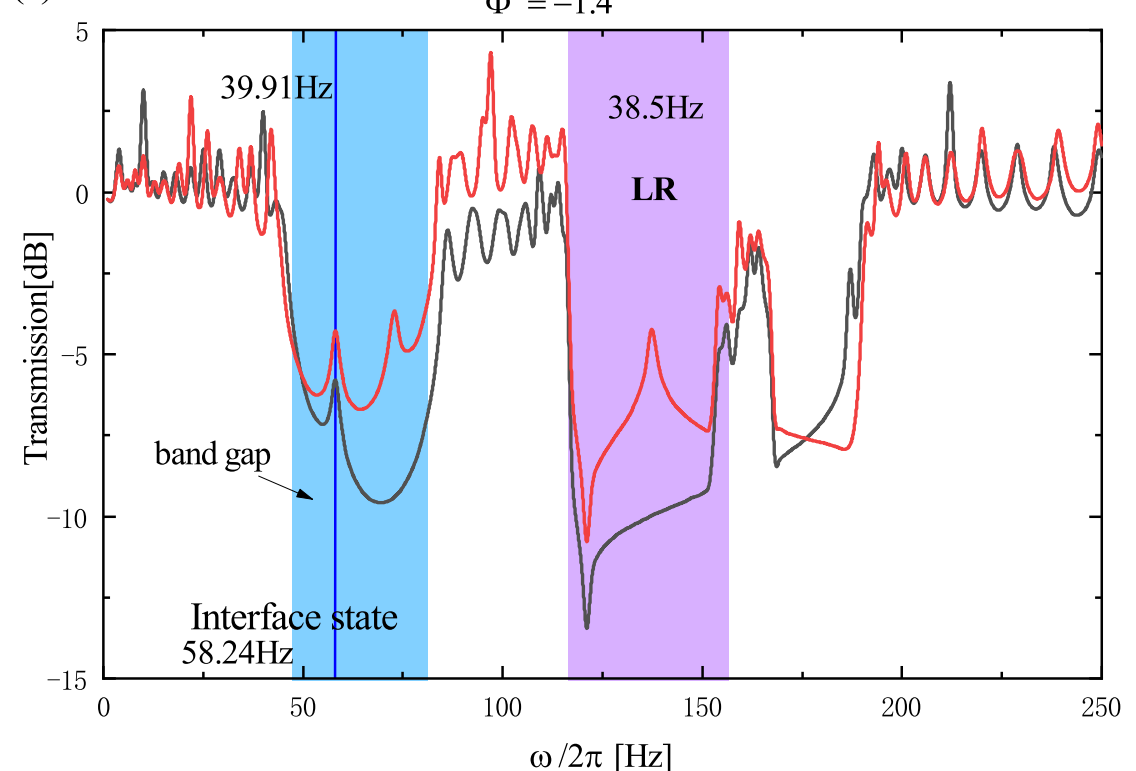

(b)

$\Phi=-1.25$

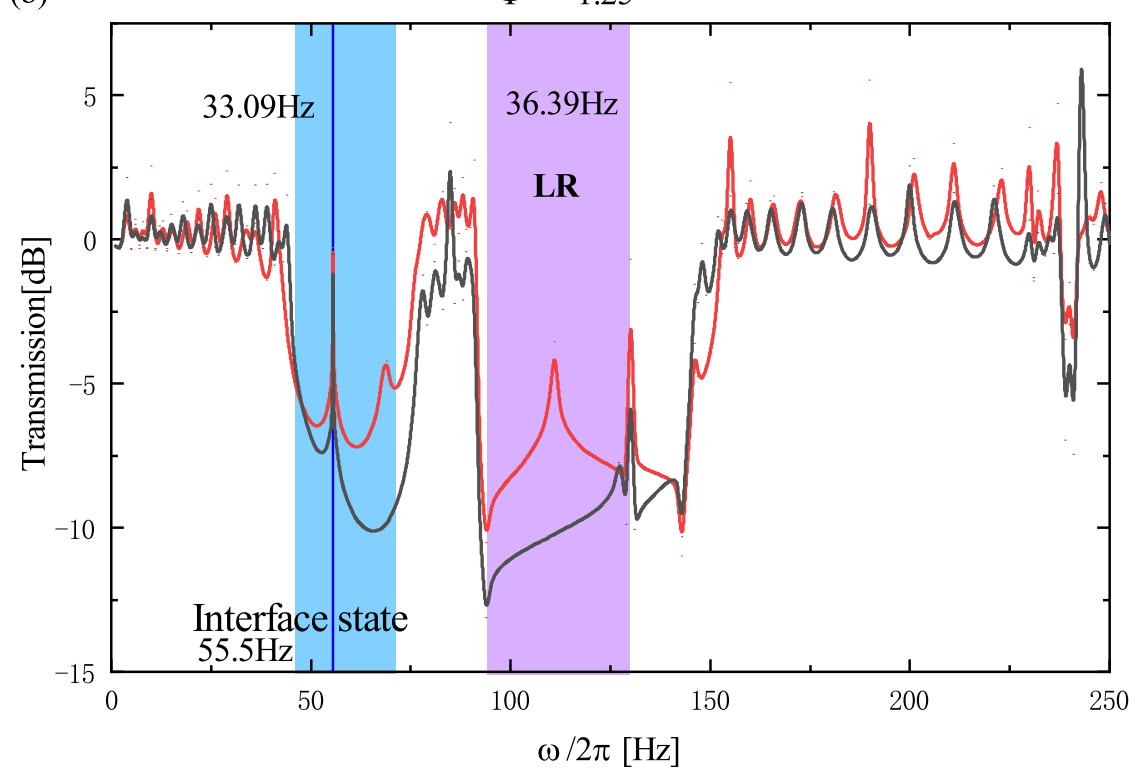

(d)

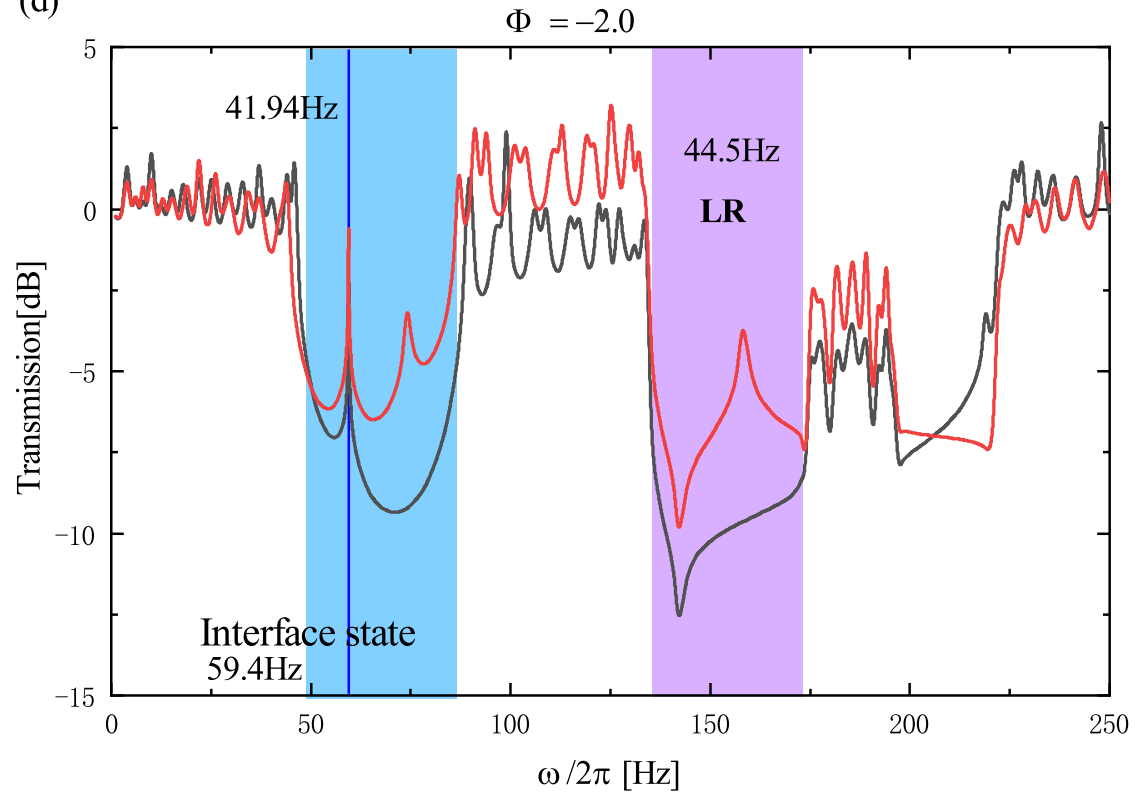


(a)

Interface mode $(58.24 \mathrm{~Hz})$

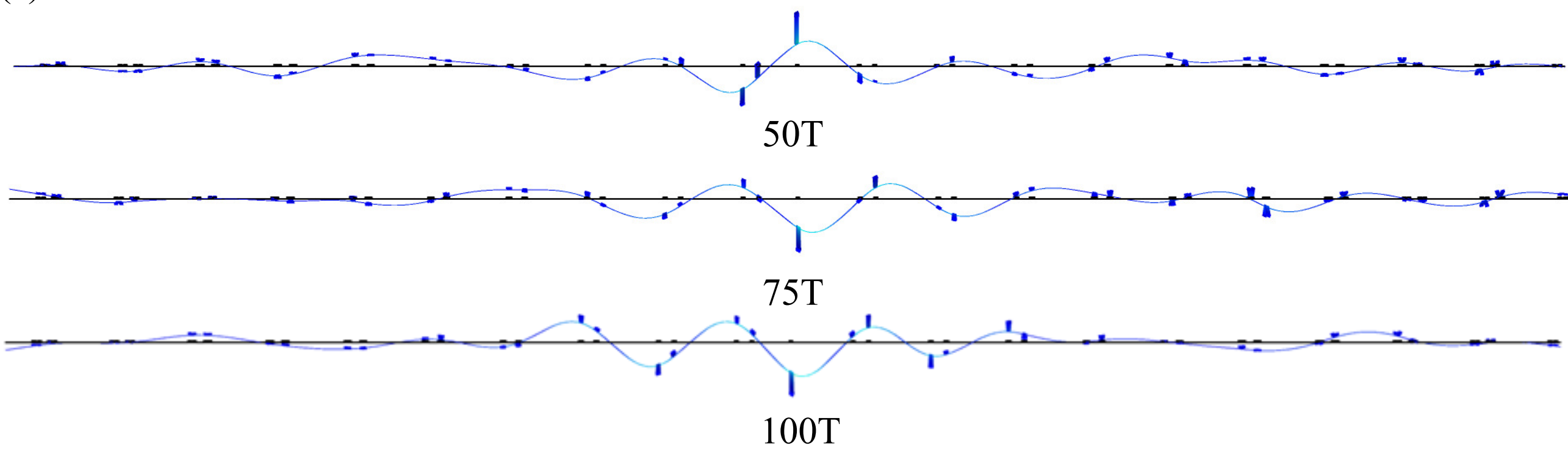

(b)

Pass band $(20 \mathrm{~Hz})$

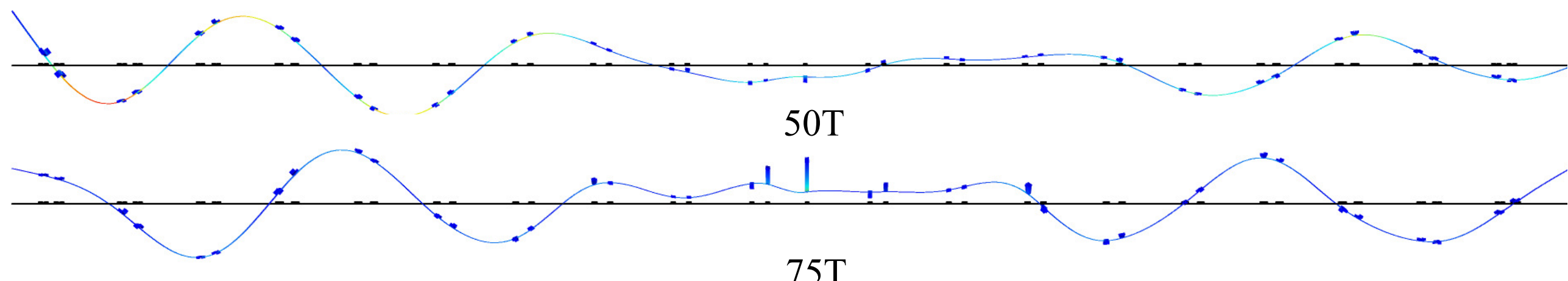

$75 \mathrm{~T}$

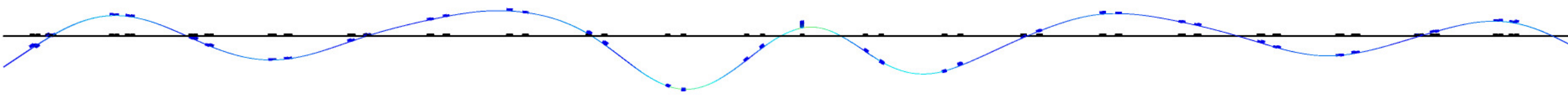

$100 \mathrm{~T}$

(c)
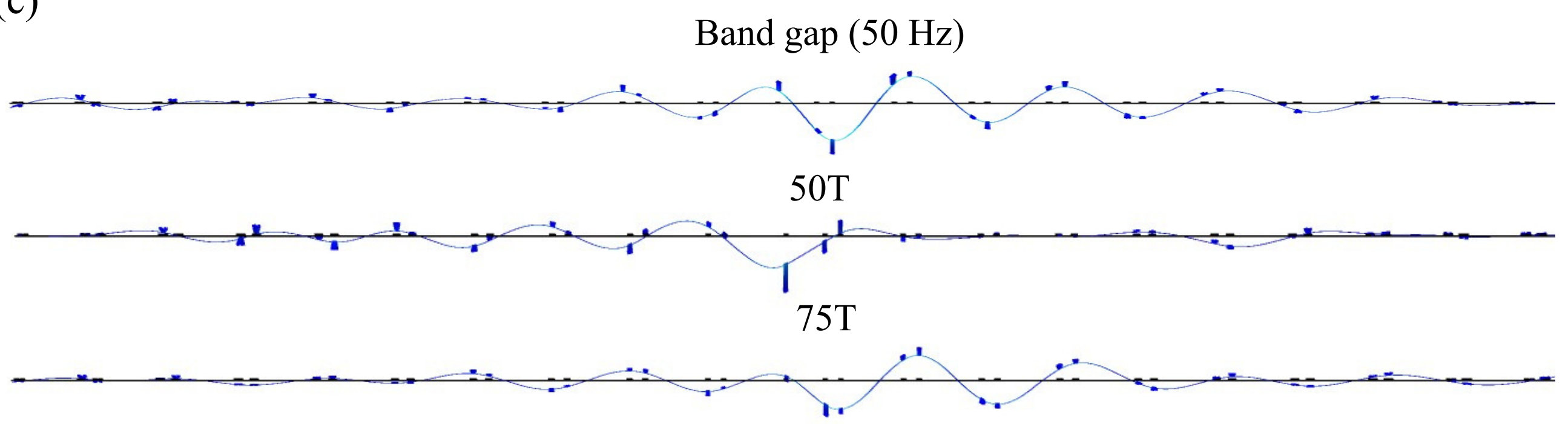

$100 \mathrm{~T}$ 


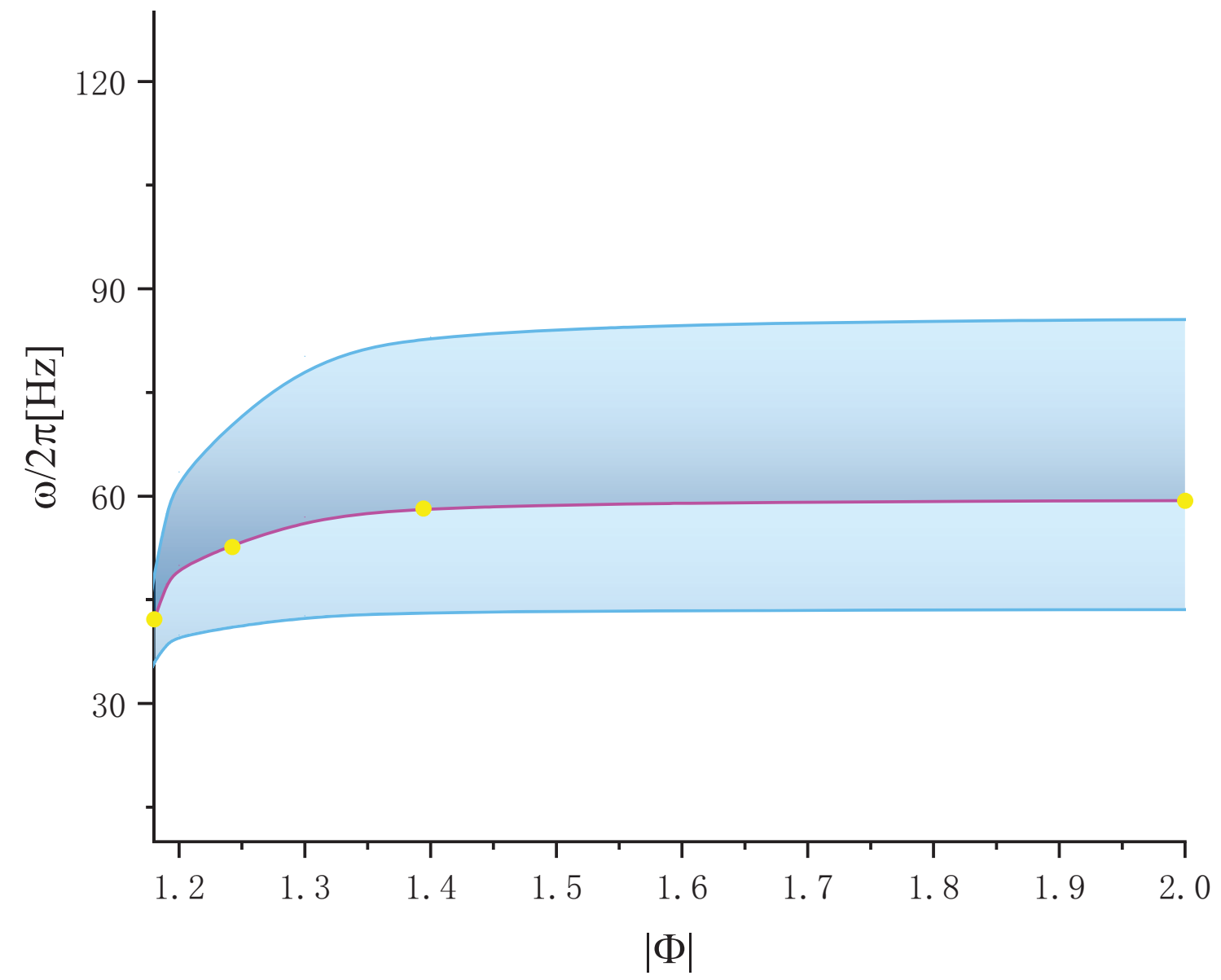




\section{Band Location}

$\beta=0.6$

$2 k l_{b}=0 \quad$ sym

1

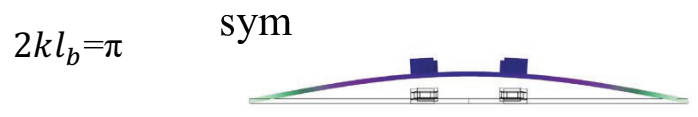

$$
2 k l_{b}=0
$$

2

$$
2 k l_{b}=\pi
$$
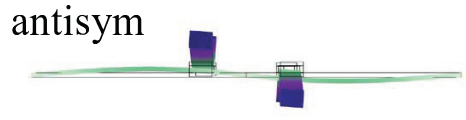

antisym sym

antisym

एकाष्म

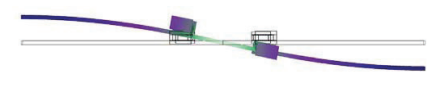

antisym

sym

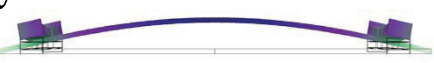

